Bull. Korean Math. Soc. 50 (2013), No. 3, pp. 839-865

http://dx.doi.org/10.4134/BKMS.2013.50.3.839

\title{
POSITIVE RADIAL SOLUTIONS FOR A CLASS OF ELLIPTIC SYSTEMS CONCENTRATING ON SPHERES WITH POTENTIAL DECAY
}

\author{
Paulo Cesar Carrião, Narciso Horta lisboa, \\ and Olimpio Hiroshi Miyagaki
}

Abstract. We deal with the existence of positive radial solutions concentrating on spheres for the following class of elliptic system

$$
\left\{\begin{array}{c}
-\varepsilon^{2} \Delta u+V_{1}(x) u=K(x) Q_{u}(u, v) \text { in } \mathbb{R}^{N}, \\
-\varepsilon^{2} \Delta v+V_{2}(x) v=K(x) Q_{v}(u, v) \text { in } \mathbb{R}^{N}, \\
u, v \in W^{1,2}\left(\mathbb{R}^{N}\right), u, v>0 \text { in } \mathbb{R}^{N},
\end{array}\right.
$$

where $\varepsilon$ is a small positive parameter; $V_{1}, V_{2} \in C^{0}\left(\mathbb{R}^{N},[0, \infty)\right)$ and $K \in C^{0}\left(\mathbb{R}^{N},(0, \infty)\right)$ are radially symmetric potentials; $Q$ is a $(p+1)$ homogeneous function and $p$ is subcritical, that is, $1<p<2^{*}-1$, where $2^{*}=2 N /(N-2)$ is the critical Sobolev exponent for $N \geq 3$.

\section{Introduction}

This work has been motivated by some papers appeared in recent years concerning the Schrödinger equation

$$
i \hbar \frac{\partial \psi}{\partial t}+\frac{\hbar^{2}}{2} \Delta \psi-V(x) \psi+K(x)|\psi|^{p-1} \psi=0, x \in \mathbb{R}^{N},
$$

where $\hbar$ denotes the Plank constant, $i$ is the imaginary unit and $p \in\left(1, \frac{N+2}{N-2}\right)$. This equation appears in many fields of physic, in particular, when we describe Bose-Einstein condensates (see [30] and [34]) and the propagation of light in some nonlinear optical material (see [35]).

For application or motivation, we can cite also, for instance, [32, 33] where are studied the evolution of two orthogonal pulse envelope in birefringent optical fibers, see also [29]. System of type $(S)$ is also important for industrial applications in fiber communications systems [27, 28]. Finally we would to recall that system of type $(S)$ can describe other physical phenomena, such

Received March 18, 2012.

2010 Mathematics Subject Classification. 35J50, 35B06, 35A15, 35B25.

Key words and phrases. Schrödinger operator, radial solution, variational method, singular perturbation.

The second author was partially supported by Fapemig/Brazil.

The third author was partially supported by CNPq/Brazil and INCTMat/Brazil. 
as Kerr-like photorefractive media in optics, (cf. [1, 20, 21, 22]), Hartree-Fock theory for double condensate [25]. See [31] and [37] for more applications in physical and chemical phenomenas.

Here we are concerned with the existence of standing waves (semiclassical states) of the nonlinear Schrödinger equations for small $\varepsilon$, that is, solutions of the form $\psi(x, t)=\exp (-i E t / \varepsilon) u(x)$. Notice that after a simple rescaling and putting $V(x)-E=V(x), \psi$ satisfies $(N L S)$ if and only if $u$ solve the elliptic equation

$(N L S)_{\varepsilon} \quad-\varepsilon^{2} \Delta u+V(x) u=K(x) u^{p}, u>0, x \in \mathbb{R}^{N}$.

The most characteristic feature of $(N L S)_{\varepsilon}$ is that its solution $u_{\varepsilon}$ concentrate as $\varepsilon \rightarrow 0$. When this concentration set is a single point (resp. finite points), these solutions are called, in the literature, spike solution (resp. multi-bump solutions). When the potential $V>0$, beginning from the pioneering paper by Floer and Weinstein [24], a great number of work has been devoted to study spike or multi-bump solutions for $(N L S)_{\varepsilon}$ (see [5] and references there in). Studying in this case $(V>0)$, Ambrosetti-Malchiodi-Ni in [6] constructed solutions concentrating on spheres for $(N L S)_{\varepsilon}$. Ambrosetti-Ruiz in [9] extended this result to the case of decaying potentials. See also [4], [7], [10], [12], [13], [15] and [23]. In the critical frequence, that means $\inf _{\mathbb{R}^{N}} V(x)=0$, spike solutions have been constructed in [16], [17], [18] and [19], which concentrate on the zero of the potential $V$ as $\varepsilon \rightarrow 0$. In those papers also are constructed "small" solutions concentrating on spheres near zeroes of the potentials. On the other hand, Alves [2] and Alves-Soares [3] studied, by using the Mountain Pass Theorem due to Ambrosetti-Rabinowitz [8], the elliptic system $(S)$, when $V_{1}$ and $V_{2}$ are globally lower bounded away from zero. The authors showed that the solution $\left(u_{\varepsilon}, v_{\varepsilon}\right)$ concentrates around local minima of the potentials $V_{1}$ and $V_{2}$.

Motivated by the above papers, we are going to construct solutions concentrating on spheres for a class of the elliptic system with decaying potentials, where $V_{1}, V_{2}$ and $K$ are radially symmetric potentials satisfying:

$(V) V_{1}, V_{2} \in C^{0}\left(\mathbb{R}^{N},[0, \infty)\right)$ are such that

$$
\liminf _{|x| \rightarrow \infty}|x|^{2} V(x) \equiv 4 \lambda>0,
$$

where $V(x)=\min \left\{V_{1}(x), V_{2}(x)\right\}$ and the zero set of $V, Z=\left\{x \in \mathbb{R}^{N}: V(x)=0\right\}$ is non-empty;

$(K) K \in C^{0}\left(\mathbb{R}^{N},(0, \infty)\right)$ is limited.

The function $Q \in C^{1}([0,+\infty) \times[0,+\infty), \mathbb{R})$ is a homogeneous function of degree $p+1$, with $1<p<\frac{N+2}{N-2}, N \geq 3$ and verify:

$\left(Q_{1}\right)$ There exists $C>0$ such that

$$
\left\{\begin{aligned}
\left|Q_{u}(u, v)\right| & \leq C\left(|u|^{p}+|v|^{p}\right), \forall u, v \geq 0 \\
\left|Q_{v}(u, v)\right| & \leq C\left(|u|^{p}+|v|^{p}\right), \forall u, v \geq 0
\end{aligned}\right.
$$


$\left(Q_{2}\right)$ There exist $\eta_{1}, \eta_{2}>0$ such that

$$
\eta_{1}\left(|u|^{p+1}+|v|^{p+1}\right) \leq Q(u, v) \leq \eta_{2}\left(|u|^{p+1}+|v|^{p+1}\right) \forall u, v>0
$$

$\left(Q_{3}\right) Q_{u}(0,1), Q_{v}(1,0)>0$;

$\left(Q_{4}\right) Q(u, v)>0 \forall u, v>0$;

$\left(Q_{5}\right) Q_{u}(u, v), Q_{v}(u, v) \geq 0 \forall u, v \geq 0$.

Remark 1. (a) Since $Q$ is a $C^{1}$ homogeneous function of degree $p+1$, then $(p+1) Q(u, v)=u Q_{u}(u, v)+v Q_{v}(u, v)$ and $\nabla Q$ is a homogeneous function of degree $p$.

(b) Note that the right hand side of $\left(Q_{2}\right)$ can be obtained from $\left(Q_{1}\right)$, (a) and the Young inequality.

(c) These kind of hypotheses were introduced for instance in [2] and [36].

(d) Our prototype of $Q$ is $Q(u, v)=(a u+b v)^{p+1}, u, v \geq 0$ and $a, b>0$.

Our main result is the following.

Theorem 1. Suppose that $\left(Q_{1}\right)-\left(Q_{5}\right),(V)$ and $(K)$ hold. Let $A \subset Z$ be an isolated compact subset of $Z$ such that $0 \notin A$ and $V_{1} \equiv V_{2}$ in $A$. Then for $\varepsilon$ sufficienthly small, $(S)$ has a solution $\left(u_{\varepsilon}, v_{\varepsilon}\right) \in W^{1,2}\left(\mathbb{R}^{N}\right) \times W^{1,2}\left(\mathbb{R}^{N}\right), u_{\varepsilon}$ and $v_{\varepsilon}$ radially symmetric functions, such that

$$
\lim _{\varepsilon \rightarrow 0}\left\|u_{\varepsilon}\right\|_{L^{\infty}\left(\mathbb{R}^{N}\right)}=\lim _{\varepsilon \rightarrow 0}\left\|v_{\varepsilon}\right\|_{L^{\infty}\left(\mathbb{R}^{N}\right)}=0
$$

and

$$
\liminf _{\varepsilon \rightarrow 0} \varepsilon^{-2 /(p-1)}\left\|u_{\varepsilon}+v_{\varepsilon}\right\|_{L^{\infty}\left(\mathbb{R}^{N}\right)}>0 .
$$

Moreover, for each $\delta>0$, there are constants $C, c>0$ such that

$$
u_{\varepsilon}(x), v_{\varepsilon}(x) \leq C \exp (-c / \varepsilon)\left[1+\left(|x| / 2 R_{0}\right)^{\omega_{\varepsilon}}\right] \forall x \in \mathbb{R}^{N} \backslash A^{4 \delta},
$$

where $\omega_{\varepsilon} \equiv-\frac{(N-2)+\sqrt{(N-2)^{2}+4 \lambda / \varepsilon^{2}}}{2}, A^{d} \equiv\left\{x \in \mathbb{R}^{N} \mid d(x, A) \leq d\right\}$ and $R_{0}$ is a positive constant given by $(V)$.

The proof of Theorem 1 is made adapting closely arguments used in [16] and [17], more exactly, the minimization techniques with two constraints in order to construct the spike solutions concentrating on sphere near of the zeros of $V_{1}$ and $V_{2}$. Actually, one of the constraints represents a type of the penalization of the nonlinearity. The proof of the decay estimate of the solution is slightly different those made in [16] and [17]. Here, in our case, we use some ideas in [11], as well as, those in [16] and [17], combining Moser iterations, classical elliptic estimates and comparison principle we obtain the decay estimate of the solutions desired. 


\section{Proof of Theorem 1}

First of all by a scaling we see that system $(S)$ is equivalent to

$$
\left\{\begin{array}{c}
-\Delta u+V_{1}(\varepsilon x) u=K(\varepsilon x) Q_{u}(u, v) \text { in } \mathbb{R}^{N}, \\
-\Delta v+V_{2}(\varepsilon x) v=K(\varepsilon x) Q_{v}(u, v) \text { in } \mathbb{R}^{N}, \\
u, v \in W^{1,2}\left(\mathbb{R}^{N}\right), u, v>0 \text { in } \mathbb{R}^{N} .
\end{array}\right.
$$

Let $A$ be the isolated compact subset of $Z$ as assumed in the theorem. We choose $\delta>0$ such that $0 \notin A^{8 \delta}$, and $A^{8 \delta} \cap(Z \backslash A)=\varnothing$, where $A^{\delta} \equiv$ $\left\{x \in \mathbb{R}^{N} \mid d(x, A) \leq \delta\right\}$. We define $A_{\varepsilon}^{\delta} \equiv\left\{x \in \mathbb{R}^{N} \mid \varepsilon x \in A^{\delta}\right\}$. Let $C_{0, \text { rad }}^{\infty}\left(\mathbb{R}^{N}\right)$ be the class of radially symmetric functions in $C_{0}^{\infty}\left(\mathbb{R}^{N}\right)$, where $C_{0}^{\infty}\left(\mathbb{R}^{N}\right)$ is the set of functions on $C^{\infty}\left(\mathbb{R}^{N}\right)$ with compact support. Let $E_{\varepsilon}$ the completion of $C_{0, \text { rad }}^{\infty}\left(\mathbb{R}^{N}\right) \times C_{0, \text { rad }}^{\infty}\left(\mathbb{R}^{N}\right)$ with respect to the norm

$$
\|(u, v)\|_{\varepsilon}=\left(\int_{\mathbb{R}^{N}}\left[|\nabla u|^{2}+|\nabla v|^{2}+V_{1}(\varepsilon x) u^{2}+V_{2}(\varepsilon x) v^{2}\right] d x\right)^{1 / 2} .
$$

We observe that $E_{\varepsilon}=E_{V_{1}, \varepsilon} \times E_{V_{2}, \varepsilon}$, where $E_{V_{i}, \varepsilon}$ is the completion of $C_{0, \text { rad }}^{\infty}\left(\mathbb{R}^{N}\right)$ with the norm $\|u\|_{V_{i}, \varepsilon}=\left(\int_{\mathbb{R}^{N}}\left[|\nabla u|^{2}+V_{i}(\varepsilon x) u^{2}\right] d x\right)^{1 / 2}, i=1,2$. Thus, $\|(u, v)\|_{\varepsilon}^{2}=\|u\|_{V_{1}, \varepsilon}^{2}+\|v\|_{V_{2}, \varepsilon}^{2}$.

We fix a constant $\gamma$ with $\gamma(p-1) /(p+1)>2$. We define a function $\chi_{\varepsilon}$ by

$$
\chi_{\varepsilon}(x)=\left\{\begin{array}{cl}
\varepsilon^{-(N-1)-3(p+1) /(p-1)} & \text { if }|x| \leq R_{0} / \varepsilon, x \notin A_{\varepsilon}^{4 \delta}, \\
(|x| / \varepsilon)^{\gamma} & \text { if }|x| \geq R_{0} / \varepsilon, \\
0 & \text { if } x \in A_{\varepsilon}^{4 \delta},
\end{array}\right.
$$

where $R_{0} \geq 1$ is fixed so that $V(x)>0$ for $|x| \geq R_{0}$ and $Z^{8 \delta} \subset B\left(0, R_{0}\right)$.

Now we consider the following minimization problem

$$
\begin{aligned}
M_{\varepsilon}=\inf \left\{\|(u, v)\|_{\varepsilon}^{2} \mid\right. & \int_{\mathbb{R}^{N}} K(\varepsilon x) Q(u, v) d x=1, \\
& \left.\int_{\mathbb{R}^{N}} \chi_{\varepsilon}(x) Q(u, v) d x \leq 1,(u, v) \in E_{\varepsilon}\right\} .
\end{aligned}
$$

First, using the same type of arguments developed in [16], we have the following lemma.

Lemma 2. $\lim _{\varepsilon \rightarrow 0} \varepsilon^{(N-1)(p-1) /(p+1)} M_{\varepsilon}=0$.

Proof. Let $x_{0} \in A$. Then, for any $a>0$, there exists $b>0$ such that $V_{1}(x), V_{2}(x) \in[0, a)$ for $\left|x-x_{0}\right| \leq b$. Without loss of generality, we can assume $\left|x_{0}\right|=1$ so that $S_{\varepsilon}^{\delta} \subset A_{\varepsilon}^{\delta}$, where $S$ is the unit sphere in $\mathbb{R}^{N}$. Then, using change of variables (polar coordenates) and setting $u(r+1 / \varepsilon)=\bar{u}(r)$, $v(r+1 / \varepsilon)=\bar{v}(r)$, we obtain that

$$
M_{\varepsilon} \leq C_{0} \frac{\int_{S_{\varepsilon}^{\delta}}\left[|\nabla u(x)|^{2}+|\nabla v(x)|^{2}+a\left((u(x))^{2}+(v(x))^{2}\right)\right] d x}{\left(\int_{S_{\varepsilon}^{\delta}} Q(u(x), v(x)) d x\right)^{2 /(p+1)}}
$$




$$
\begin{aligned}
\leq & C \varepsilon^{-(N-1)(p-1) /(p+1)} \\
& \times \frac{\int_{-\delta / \varepsilon}^{\delta / \varepsilon}\left[\left(\bar{u}^{\prime}(r)\right)^{2}+\left(\bar{v}^{\prime}(r)\right)^{2}+a\left((\bar{u}(r))^{2}+(\bar{v}(r))^{2}\right)\right] d r}{\left(\int_{-\delta / \varepsilon}^{\delta / \varepsilon} Q(\bar{u}(r), \bar{v}(r)) d r\right)^{2 /(p+1)}},
\end{aligned}
$$

where $C_{0}$ and $C$ are positive constants independent of $\varepsilon$. Here was used that $\chi_{\varepsilon}(x)=0, \forall x \in S_{\varepsilon}^{\delta} ; V_{1}$ and $V_{2}$ are radially symmetric, and $V_{1}(\varepsilon x), V_{2}(\varepsilon x)<a$, $\forall x \in S_{\varepsilon}^{\delta}$. Setting $\bar{u}(r)=u(\sqrt{a} r)$ and $\bar{v}(r)=v(\sqrt{a} r)$, where $a>0$ is arbitrary, we obtain,

$$
\begin{aligned}
& \lim _{\varepsilon \rightarrow 0} \varepsilon^{(N-1)(p-1) /(p+1)} M_{\varepsilon} \\
\leq & C a^{(p+3) / 2(p+1)} \inf _{u, v \in C_{0}^{\infty}(-\infty, \infty)} \frac{\int_{-\infty}^{\infty}\left[\left(u^{\prime}\right)^{2}+\left(v^{\prime}\right)^{2}+u^{2}+v^{2}\right] d r}{\left(\int_{-\infty}^{\infty} Q(u, v) d r\right)^{2 /(p+1)}} .
\end{aligned}
$$

Then, since $a$ is arbitrary and the last infimun is bounded, the lemma follows.

Lemma 3. For sufficiently small $\varepsilon>0, M_{\varepsilon}$ is achieved at $\left(\bar{u}_{\varepsilon}, \bar{v}_{\varepsilon}\right) \in E_{\varepsilon}$ which satisfies for some $\alpha_{\varepsilon}>0 \geq \beta_{\varepsilon}$,

$$
\left\{\begin{array}{cll}
\left(S_{\alpha_{\varepsilon}}, \beta \varepsilon\right) & & \\
-\Delta \bar{u}_{\varepsilon}+V_{1}(\varepsilon x) \bar{u}_{\varepsilon}=\alpha_{\varepsilon} K(\varepsilon x) Q_{u}\left(\bar{u}_{\varepsilon}, \bar{v}_{\varepsilon}\right)+\beta_{\varepsilon} \chi_{\varepsilon}(x) Q_{u}\left(\bar{u}_{\varepsilon}, \bar{v}_{\varepsilon}\right) & \text { in } & \mathbb{R}^{N}, \\
-\Delta \bar{v}_{\varepsilon}+V_{2}(\varepsilon x) \bar{v}_{\varepsilon}=\alpha_{\varepsilon} K(\varepsilon x) Q_{v}\left(\bar{u}_{\varepsilon}, \bar{v}_{\varepsilon}\right)+\beta_{\varepsilon} \chi_{\varepsilon}(x) Q_{v}\left(\bar{u}_{\varepsilon}, \bar{v}_{\varepsilon}\right) & \text { in } \mathbb{R}^{N} \\
\bar{u}_{\varepsilon} \geq 0, \bar{v}_{\varepsilon} \geq 0 & \text { in } \mathbb{R}^{N}
\end{array}\right.
$$

Proof. Let $\left\{\left(\bar{u}_{\varepsilon}^{j}, \bar{v}_{\varepsilon}^{j}\right)\right\}_{j} \subset E_{\varepsilon}$ be a minimizing sequence for $M_{\varepsilon}$. We can assume $\left\{\left(\bar{u}_{\varepsilon}^{j}, \bar{v}_{\varepsilon}^{j}\right)\right\}_{j} \subset C_{0, \text { rad }}^{\infty}\left(\mathbb{R}^{N}\right) \times C_{0, \text { rad }}^{\infty}\left(\mathbb{R}^{N}\right)$, since $C_{0, \text { rad }}^{\infty}\left(\mathbb{R}^{N}\right) \times C_{0, \text { rad }}^{\infty}\left(\mathbb{R}^{N}\right)$ is dense in $E_{\varepsilon}$. We take $R_{j}>0$ such that $\operatorname{supp}\left(\bar{u}_{\varepsilon}^{j}\right) \subset B\left(0, R_{j}\right)$ and $\operatorname{supp}\left(\bar{v}_{\varepsilon}^{j}\right) \subset B\left(0, R_{j}\right)$, $j \geq 1$. For a fixed $\varepsilon>0$, we can assume that $R_{0} / \varepsilon<R_{1}<R_{2}<\cdots$ and $\lim _{m \rightarrow \infty} R_{m}=\infty$. We define

$$
E_{\varepsilon}^{m} \equiv E_{\varepsilon} \cap\left(W_{0}^{1,2}\left(B\left(0, R_{m}\right)\right) \times W_{0}^{1,2}\left(B\left(0, R_{m}\right)\right)\right) .
$$

We consider a restricted minimization problem

$$
\begin{aligned}
M_{\varepsilon}^{m}=\inf \left\{\|(u, v)\|_{\varepsilon}^{2} \mid\right. & \int_{\mathbb{R}^{N}} K(\varepsilon x) Q(u, v) d x=1 \\
& \left.\int_{\mathbb{R}^{N}} \chi_{\varepsilon}(x) Q(u, v) d x \leq 1,(u, v) \in E_{\varepsilon}^{m}\right\} .
\end{aligned}
$$

Now, we will prove that there exists a non-negative minimizer $\left(u_{\varepsilon}^{m}, v_{\varepsilon}^{m}\right)$ of $M_{\varepsilon}^{m}$ such that $M_{\varepsilon} \leq M_{\varepsilon}^{m}$ and $\lim _{m \rightarrow \infty} M_{\varepsilon}^{m}=M_{\varepsilon}$. Indeed, let $\left\{\left(u_{\varepsilon}^{k}, v_{\varepsilon}^{k}\right)\right\}_{k}$ be a minimizing sequence for $M_{\varepsilon}^{m}$. Then it follows that $\left\{\left(u_{\varepsilon}^{k}, v_{\varepsilon}^{k}\right)\right\}_{k}$ is bounded. Since $E_{\varepsilon}^{m}$ is reflexive, there exists $\left(u_{\varepsilon}^{m}, v_{\varepsilon}^{m}\right) \in E_{\varepsilon}^{m}$ such that $\left\{\left(u_{\varepsilon}^{k}, v_{\varepsilon}^{k}\right)\right\}_{k}$ is weakly convergent to $\left(u_{\varepsilon}^{m}, v_{\varepsilon}^{m}\right)$, up to subsequence. Thus, $u_{\varepsilon}^{k} \rightarrow u_{\varepsilon}^{m}$ weakly in $E_{V_{1}, \varepsilon}$ and $v_{\varepsilon}^{k} \rightarrow v_{\varepsilon}^{m}$ weakly in $E_{V_{2}, \varepsilon}$ as $k \rightarrow \infty$. Since $E_{V_{i}, \varepsilon} \cap W_{0}^{1,2} B\left(\left(0, R_{m}\right)\right)$ 
is compactly imbedded in $L^{p+1}\left(B\left(0, R_{m}\right)\right)$, with $i=1,2$ and $2<p+1<2^{*}$, from $\left(Q_{2}\right)$ we have

$$
\int_{B\left(0, R_{m}\right)} K(\varepsilon x) Q\left(u_{\varepsilon}^{m}, v_{\varepsilon}^{m}\right) d x=\lim _{k \rightarrow \infty} \int_{B\left(0, R_{m}\right)} K(\varepsilon x) Q\left(u_{\varepsilon}^{k}, v_{\varepsilon}^{k}\right) d x=1,
$$

and

$$
\int_{B\left(0, R_{m}\right)} \chi_{\varepsilon} Q\left(u_{\varepsilon}^{m}, v_{\varepsilon}^{m}\right) d x=\lim _{k \rightarrow \infty} \int_{B\left(0, R_{m}\right)} \chi_{\varepsilon} Q\left(u_{\varepsilon}^{k}, v_{\varepsilon}^{k}\right) d x \leq 1 .
$$

Since $\left\{\left(u_{\varepsilon}^{k}, v_{\varepsilon}^{k}\right)\right\}_{k}$ is weakly convergent to $\left(u_{\varepsilon}^{m}, v_{\varepsilon}^{m}\right)$, we have

$$
\left\|\left(u_{\varepsilon}^{m}, v_{\varepsilon}^{m}\right)\right\|_{\varepsilon}^{2} \leq \liminf _{k \rightarrow \infty}\left\|\left(u_{\varepsilon}^{k}, v_{\varepsilon}^{k}\right)\right\|_{\varepsilon}^{2}=M_{\varepsilon}^{m} \leq\left\|\left(u_{\varepsilon}^{m}, v_{\varepsilon}^{m}\right)\right\|_{\varepsilon}^{2} .
$$

Thus, $\left(u_{\varepsilon}^{m}, v_{\varepsilon}^{m}\right)$ is a minimizer for $M_{\varepsilon}^{m}$. Since $|\nabla| u_{\varepsilon}^{m}||=\left|\nabla u_{\varepsilon}^{m}\right|$ and $|\nabla| v_{\varepsilon}^{m}||=$ $\left|\nabla v_{\varepsilon}^{m}\right|$ we see that $\left\|\left(u_{\varepsilon}^{m}, v_{\varepsilon}^{m}\right)\right\|_{\varepsilon}^{2}=\left\|\left(\left|u_{\varepsilon}^{m}\right|,\left|v_{\varepsilon}^{m}\right|\right)\right\|_{\varepsilon}^{2}$. Then, there exists a nonnegative minimizer $\left(u_{\varepsilon}^{m}, v_{\varepsilon}^{m}\right)$ of $M_{\varepsilon}^{m}$. Now, we observe that for any $j \geq 1$,

$$
\lim _{k \rightarrow \infty}\left\|\left(u_{\varepsilon}^{k}, v_{\varepsilon}^{k}\right)\right\|_{\varepsilon}^{2} \leq\left\|\left(u_{\varepsilon}^{j}, v_{\varepsilon}^{j}\right)\right\|_{\varepsilon}^{2} .
$$

In fact, for any $j \leq k, B\left(0, R_{j}\right) \subset B\left(0, R_{k}\right)$. Thus,

$$
W_{0}^{1,2}\left(B\left(0, R_{j}\right)\right) \subset W_{0}^{1,2}\left(B\left(0, R_{k}\right)\right) .
$$

Consequently, $E_{\varepsilon}^{j} \subset E_{\varepsilon}^{k}$. This implies that $M_{\varepsilon}^{j}=\left\|\left(u_{\varepsilon}^{j}, v_{\varepsilon}^{j}\right)\right\|_{\varepsilon}^{2} \geq\left\|\left(u_{\varepsilon}^{k}, v_{\varepsilon}^{k}\right)\right\|_{\varepsilon}^{2}=$ $M_{\varepsilon}^{k}$. We note that

$$
M_{\varepsilon} \leq \lim _{j \rightarrow \infty} M_{\varepsilon}^{j}=\lim _{j \rightarrow \infty}\left\|\left(u_{\varepsilon}^{j}, v_{\varepsilon}^{j}\right)\right\|_{\varepsilon}^{2} \leq \lim _{j \rightarrow \infty}\left\|\left(\bar{u}_{\varepsilon}^{j}, \bar{v}_{\varepsilon}^{j}\right)\right\|_{\varepsilon}^{2}=M_{\varepsilon} .
$$

Therefore, $M_{\varepsilon}^{m} \rightarrow M_{\varepsilon}$ as $m \rightarrow \infty$. Thus $\left\{\left(u_{\varepsilon}^{m}, v_{\varepsilon}^{m}\right)\right\}_{m}$ is a minimizing sequence for $M_{\varepsilon}$.

Since $\left(u_{\varepsilon}^{m}, v_{\varepsilon}^{m}\right)$ is a minimizer for $M_{\varepsilon}^{m}$, there exist Lagrange multipliers $\alpha_{\varepsilon}^{m}$, $\beta_{\varepsilon}^{m} \in \mathbb{R}$ such that $\left(u_{\varepsilon}^{m}, v_{\varepsilon}^{m}\right)$ satisfies the system $\left(S_{\alpha_{\varepsilon}^{m}, \beta_{\varepsilon}^{m}}\right)$ in $B\left(0, R_{m}\right)$. Taking a subsequence if necessary, we can assume that for some $\left(\bar{u}_{\varepsilon}, \bar{v}_{\varepsilon}\right) \in E_{\varepsilon}$, $\left\{\left(u_{\varepsilon}^{m}, v_{\varepsilon}^{m}\right)\right\}_{m}$ converges weakly to $\left(\bar{u}_{\varepsilon}, \bar{v}_{\varepsilon}\right)$ in $E_{\varepsilon}$ as $m \rightarrow \infty$. Since

$$
\int_{\mathbb{R}^{N}} \chi_{\varepsilon} Q\left(u_{\varepsilon}^{m}, v_{\varepsilon}^{m}\right) d x \leq 1,
$$

it follows that for any $R \geq \frac{R_{0}}{\varepsilon}$,

$$
\int_{\mathbb{R}^{N} \backslash B(0, R)} K(\varepsilon x) Q\left(u_{\varepsilon}^{m}, v_{\varepsilon}^{m}\right) d x \leq C(\varepsilon / R)^{\gamma}
$$

for some $C>0$. By the Dominated Convergence Theorem of Lebesgue, we obtain $\int_{B(0, R)} K(\varepsilon x) Q\left(\bar{u}_{\varepsilon}, \bar{v}_{\varepsilon}\right) d x \geq 1-C(\varepsilon / R)^{\gamma}$. This implies that

$$
\int_{\mathbb{R}^{N}} K(\varepsilon x) Q\left(\bar{u}_{\varepsilon}, \bar{v}_{\varepsilon}\right) d x=\lim _{R \rightarrow \infty} \int_{B(0, R)} K(\varepsilon x) Q\left(\bar{u}_{\varepsilon}, \bar{v}_{\varepsilon}\right) d x \geq 1 .
$$


We claim that

$$
\int_{\mathbb{R}^{N}} K(\varepsilon x) Q\left(\bar{u}_{\varepsilon}, \bar{v}_{\varepsilon}\right) d x=1 .
$$

In fact, arguing by contradiction, we assume that $\int_{\mathbb{R}^{N}} K(\varepsilon x) Q\left(\bar{u}_{\varepsilon}, \bar{v}_{\varepsilon}\right) d x>1$. Then there exists $\bar{R}>0$ so that $\int_{B(0, \bar{R})} K(\varepsilon x) Q\left(\bar{u}_{\varepsilon}, \bar{v}_{\varepsilon}\right) d x>1$. Hence, we get $\lim _{m \rightarrow \infty} \int_{B(0, \bar{R})} K(\varepsilon x) Q\left(u_{\varepsilon}^{m}, v_{\varepsilon}^{m}\right) d x=\int_{B(0, \bar{R})} K(\varepsilon x) Q\left(\bar{u}_{\varepsilon}, \bar{v}_{\varepsilon}\right) d x>1$. Thus, there exists $m_{0} \in \mathbb{N}$ such that $\int_{B(0, \bar{R})} K(\varepsilon x) Q\left(u_{\varepsilon}^{m_{0}}, v_{\varepsilon}^{m_{0}}\right) d x>1$. But this is impossible, since $\int_{\mathbb{R}^{N}} K(\varepsilon x) Q\left(u_{\varepsilon}^{m}, v_{\varepsilon}^{m}\right) d x=1$ for all $m \in \mathbb{N}$.

Since $\int_{B(0, T)} \chi_{\varepsilon} Q\left(u_{\varepsilon}^{m}, v_{\varepsilon}^{m}\right) d x \leq 1$ for each $T>0$ we get, again using the Dominated Convergence Theorem of Lebesgue, that

$$
\int_{B(0, T)} \chi_{\varepsilon} Q\left(\bar{u}_{\varepsilon}, \bar{v}_{\varepsilon}\right) d x \leq 1
$$

for each $T>0$. Consequently,

$$
\int_{\mathbb{R}^{N}} \chi_{\varepsilon} Q\left(\bar{u}_{\varepsilon}, \bar{v}_{\varepsilon}\right) d x \leq 1
$$

Since $\left\|\left(\bar{u}_{\varepsilon}, \bar{v}_{\varepsilon}\right)\right\|_{\varepsilon}^{2} \leq \liminf _{m \rightarrow \infty}\left\|\left(u_{\varepsilon}^{m}, v_{\varepsilon}^{m}\right)\right\|_{\varepsilon}^{2}=M_{\varepsilon}$, we infer that $\left(\bar{u}_{\varepsilon}, \bar{v}_{\varepsilon}\right)$ is a minimizer of $M_{\varepsilon}$.

Now, we will prove that in system $\left(S_{\alpha_{\varepsilon}^{m}}, \beta_{\varepsilon}^{m}\right), \alpha_{\varepsilon}^{m}>0 \geq \beta_{\varepsilon}^{m}$. In fact, using same ideas in [14], we take $\xi_{0}, \xi_{1} \in C_{0}^{\infty}\left(\mathbb{R}^{N}\right)$ non-negative radially symmetric functions with $\operatorname{supp}\left(\xi_{0}\right) \subset \operatorname{int}\left(A_{\varepsilon}^{4 \delta}\right)$ and $\operatorname{supp}\left(\xi_{1}\right) \subset\left\{x \in \mathbb{R}^{N}|| x \mid<d\left(0, A_{\varepsilon}^{4 \delta}\right)\right\}$. Define

$$
D(s, t) \equiv \int_{B\left(0, R_{m}\right)} K(\varepsilon x) Q\left(\left(1+t \xi_{0}-s \xi_{1}\right)\left(u_{\varepsilon}^{m}, v_{\varepsilon}^{m}\right)\right) d x .
$$

The function $D$ is continuously differentiable in a neighborhood of $(0,0)$. We note that $D(0,0)=1$ and $\frac{\partial}{\partial t} D(0,0)=(p+1) \int_{B\left(0, R_{m}\right)} K(\varepsilon x) \xi_{0} Q\left(u_{\varepsilon}^{m}, v_{\varepsilon}^{m}\right) d x>$ 0 . By the implicit function theorem, for small $\tau>0$ there exists $t \in C^{1}(-\tau, \tau)$ such that

$$
t(0)=0 \text { and } D(s, t(s))=1 \text { for all } s \in(-\tau, \tau)
$$

Hence

$$
(p+1) \int_{B\left(0, R_{m}\right)} K(\varepsilon x)\left(t^{\prime}(0) \xi_{0}-\xi_{1}\right) Q\left(u_{\varepsilon}^{m}, v_{\varepsilon}^{m}\right) d x=0 .
$$

Moreover, using the definition of $\chi_{\varepsilon}$ and the fact that $\chi_{\varepsilon} \xi_{0} \equiv 0$ in $B\left(0, R_{m}\right)$, we obtain

$$
\begin{aligned}
& \left.\frac{d}{d s}\right|_{s=0} \int_{B\left(0, R_{m}\right)} \chi_{\varepsilon} Q\left(\left(1+t(s) \xi_{0}-s \xi_{1}\right)\left(u_{\varepsilon}^{m}, v_{\varepsilon}^{m}\right)\right) d x \\
= & -(p+1) \varepsilon^{-(N-1)-3(p+1) /(p-1)} \int_{\operatorname{supp}\left(\xi_{1}\right)} \xi_{1} Q\left(u_{\varepsilon}^{m}, v_{\varepsilon}^{m}\right) d x<0 .
\end{aligned}
$$


This implies that there exists $c>0$ such that for any $s \in(0, c)$,

$$
\int_{B\left(0, R_{m}\right)} \chi_{\varepsilon} Q\left(\left(1+t(s) \xi_{0}-s \xi_{1}\right)\left(u_{\varepsilon}^{m}, v_{\varepsilon}^{m}\right)\right) d x<1 .
$$

Since $\left(u_{\varepsilon}^{m}, v_{\varepsilon}^{m}\right)$ is a minimizer for $M_{\varepsilon}^{m}$, we have

$$
\begin{aligned}
0 \leq & \left.\frac{d}{d s}\right|_{s=0} \int_{B\left(0, R_{m}\right)}\left[\left|\nabla\left(\left(1+t(s) \xi_{0}-s \xi_{1}\right) u_{\varepsilon}^{m}\right)\right|^{2}+\left|\nabla\left(\left(1+t(s) \xi_{0}-s \xi_{1}\right) v_{\varepsilon}^{m}\right)\right|^{2}\right. \\
& \left.+\left(1+t(s) \xi_{0}-s \xi_{1}\right)^{2}\left(V_{1}(\varepsilon x)\left(u_{\varepsilon}^{m}\right)^{2}+V_{2}(\varepsilon x)\left(v_{\varepsilon}^{m}\right)^{2}\right)\right] d x \\
= & 2 \int_{B\left(0, R_{m}\right)}\left[\nabla u_{\varepsilon}^{m} \cdot \nabla\left(\left(t^{\prime}(0) \xi_{0}-\xi_{1}\right) u_{\varepsilon}^{m}\right)+\nabla v_{\varepsilon}^{m} \cdot \nabla\left(\left(t^{\prime}(0) \xi_{0}-\xi_{1}\right) v_{\varepsilon}^{m}\right)\right. \\
& \left.+\left(t^{\prime}(0) \xi_{0}-\xi_{1}\right)\left(V_{1}(\varepsilon x)\left(u_{\varepsilon}^{m}\right)^{2}+V_{2}(\varepsilon x)\left(v_{\varepsilon}^{m}\right)^{2}\right)\right] d x .
\end{aligned}
$$

Using $\left(t^{\prime}(0) \xi_{0}-\xi_{1}\right)\left(u_{\varepsilon}^{m}, v_{\varepsilon}^{m}\right)$ as test function in $\left(S_{\alpha_{\varepsilon}^{m}, \beta_{\varepsilon}^{m}}\right)$, the homogeneity of $Q$, the definition of $\chi_{\varepsilon}$ and (9), we deduce that

$$
\begin{aligned}
0 \leq & \int_{B\left(0, R_{m}\right)}\left[\nabla u_{\varepsilon}^{m} \cdot \nabla\left(\left(t^{\prime}(0) \xi_{0}-\xi_{1}\right) u_{\varepsilon}^{m}\right)+\nabla v_{\varepsilon}^{m} \cdot \nabla\left(\left(t^{\prime}(0) \xi_{0}-\xi_{1}\right) v_{\varepsilon}^{m}\right)\right. \\
& \left.+\left(t^{\prime}(0) \xi_{0}-\xi_{1}\right)\left(V_{1}(\varepsilon x)\left(u_{\varepsilon}^{m}\right)^{2}+V_{2}(\varepsilon x)\left(v_{\varepsilon}^{m}\right)^{2}\right)\right] d x \\
= & (p+1) \alpha_{\varepsilon}^{m} \int_{B\left(0, R_{m}\right)}\left(t^{\prime}(0) \xi_{0}-\xi_{1}\right) K(\varepsilon x) Q\left(u_{\varepsilon}^{m}, v_{\varepsilon}^{m}\right) d x \\
& +(p+1) \beta_{\varepsilon}^{m} \int_{B\left(0, R_{m}\right)} \chi_{\varepsilon}\left(t^{\prime}(0) \xi_{0}-\xi_{1}\right) Q\left(u_{\varepsilon}^{m}, v_{\varepsilon}^{m}\right) d x \\
= & -(p+1) \beta_{\varepsilon}^{m} \varepsilon^{-(N-1)-3(p+1) /(p-1)} \int_{\operatorname{supp}\left(\xi_{1}\right)} \xi_{1} Q\left(u_{\varepsilon}^{m}, v_{\varepsilon}^{m}\right) d x .
\end{aligned}
$$

By (10) and (11) we conclude that $\beta_{\varepsilon}^{m} \leq 0$.

Now, taking $\left(u_{\varepsilon}^{m}, v_{\varepsilon}^{m}\right)$ as test function in $\left(S_{\alpha_{\varepsilon}^{m}}, \beta_{\varepsilon}^{m}\right)$ and using (6) we obtain

$$
\left\|\left(u_{\varepsilon}^{m}, v_{\varepsilon}^{m}\right)\right\|_{\varepsilon}^{2}=(p+1) \alpha_{\varepsilon}^{m}+(p+1) \beta_{\varepsilon}^{m} \int_{B\left(0, R_{m}\right)} \chi_{\varepsilon} Q\left(u_{\varepsilon}^{m}, v_{\varepsilon}^{m}\right) d x .
$$

This implies that $\alpha_{\varepsilon}^{m}>0$.

Now we will show that $\left(\bar{u}_{\varepsilon}, \bar{v}_{\varepsilon}\right)$ satisfies the system $\left(S_{\alpha_{\varepsilon}, \beta_{\varepsilon}}\right)$. We claim that $\left\{\alpha_{\varepsilon}^{m}\right\}_{m}$ is bounded for small $\varepsilon>0$. Indeed, arguing by contradiction assume, without loss of generality, that $\lim _{m \rightarrow \infty} \alpha_{\varepsilon}^{m}=\infty$. For any $\sigma>0$, choose a function $\phi_{\sigma} \in C_{0}^{\infty}\left(\operatorname{int}\left(A_{\varepsilon}^{4 \delta}\right)\right)$ such that $0 \leq \phi_{\sigma} \leq 1, \phi_{\sigma}(x)=1$ for $d\left(x, \partial A_{\varepsilon}^{4 \delta}\right) \geq \sigma$, and $\left|\nabla \phi_{\sigma}\right| \leq 2 / \sigma$. Using $\phi_{\sigma}\left(u_{\varepsilon}^{m}, v_{\varepsilon}^{m}\right)$ as test function in $\left(S_{\alpha_{\varepsilon}^{m}, \beta_{\varepsilon}^{m}}\right)$ and that $\chi_{\varepsilon} \phi_{\sigma} \equiv 0$, we obtain

$$
\begin{aligned}
& \int_{\mathbb{R}^{N}}\left[\left|\nabla u_{\varepsilon}^{m}\right|^{2} \phi_{\sigma}+\nabla u_{\varepsilon}^{m} \cdot \nabla \phi_{\sigma} u_{\varepsilon}^{m}+\left|\nabla v_{\varepsilon}^{m}\right|^{2} \phi_{\sigma}+\nabla v_{\varepsilon}^{m} \cdot \nabla \phi_{\sigma} v_{\varepsilon}^{m}\right. \\
& \left.+\phi_{\sigma}\left(V_{1}(\varepsilon x)\left(u_{\varepsilon}^{m}\right)^{2}+V_{2}(\varepsilon x)\left(v_{\varepsilon}^{m}\right)^{2}\right)\right] d x
\end{aligned}
$$




$$
=(p+1) \alpha_{\varepsilon}^{m} \int_{\mathbb{R}^{N}} K(\varepsilon x) Q\left(u_{\varepsilon}^{m}, v_{\varepsilon}^{m}\right) \phi_{\sigma} d x .
$$

From $\inf _{x \in \operatorname{supp}\left(\left|\nabla \phi_{\sigma}\right|\right)} V(\varepsilon x)>0$ and the properties of $\phi_{\sigma}$, we have

$$
\begin{aligned}
& \int_{\mathbb{R}^{N}}\left[\left|\nabla u_{\varepsilon}^{m}\right|^{2} \phi_{\sigma}+\nabla u_{\varepsilon}^{m} \cdot \nabla \phi_{\sigma} u_{\varepsilon}^{m}+\left|\nabla v_{\varepsilon}^{m}\right|^{2} \phi_{\sigma}+\nabla v_{\varepsilon}^{m} \cdot \nabla \phi_{\sigma} v_{\varepsilon}^{m}\right. \\
& \left.+\phi_{\sigma}\left(V_{1}(\varepsilon x)\left(u_{\varepsilon}^{m}\right)^{2}+V_{2}(\varepsilon x)\left(v_{\varepsilon}^{m}\right)^{2}\right)\right] d x \\
\leq & C\left\|\left(u_{\varepsilon}^{m}, v_{\varepsilon}^{m}\right)\right\|_{\varepsilon}^{2}
\end{aligned}
$$

for some $C>0$, independent of $m$. By (13), (14) and the fact that

$$
\left\{\left\|\left(u_{\varepsilon}^{m}, v_{\varepsilon}^{m}\right)\right\|_{\varepsilon}^{2}\right\}_{m}
$$

is a bounded sequence, we see that for some $C>0$, independent of $m$,

$$
\int_{\mathbb{R}^{N}} K(\varepsilon x) Q\left(u_{\varepsilon}^{m}, v_{\varepsilon}^{m}\right) \phi_{\sigma} d x \leq C / \alpha_{\varepsilon}^{m} .
$$

Thus,

$$
\lim _{m \rightarrow \infty} \int_{\left\{x \in A_{\varepsilon}^{4 \delta} \mid d\left(x, \partial A_{\varepsilon}^{4 \delta}\right) \geq \sigma\right\}} K(\varepsilon x) Q\left(u_{\varepsilon}^{m}, v_{\varepsilon}^{m}\right) d x=0 .
$$

From the condition $\int_{\mathbb{R}^{N}} \chi_{\varepsilon} Q\left(u_{\varepsilon}^{m}, v_{\varepsilon}^{m}\right) d x \leq 1$ and from the definition of $\chi_{\varepsilon}$, we have

$$
\int_{\mathbb{R}^{N} \backslash B\left(0, R_{0} / \varepsilon\right)} K(\varepsilon x) Q\left(u_{\varepsilon}^{m}, v_{\varepsilon}^{m}\right) d x \leq C\left(\varepsilon / R_{0}\right)^{\gamma}
$$

and

$$
\int_{B\left(0, R_{0} / \varepsilon\right) \backslash A_{\varepsilon}^{4 \delta}} K(\varepsilon x) Q\left(u_{\varepsilon}^{m}, v_{\varepsilon}^{m}\right) d x \leq C \varepsilon^{(N-1)+3(p+1) /(p-1)}
$$

for some positive constant $C$. Now, using $\int_{\mathbb{R}^{N}} K(\varepsilon x) Q\left(u_{\varepsilon}^{m}, v_{\varepsilon}^{m}\right) d x=1$, (15), (16) and (17) we infer that

$$
\begin{aligned}
& \liminf _{m \rightarrow \infty} \int_{\left\{x \in A_{\varepsilon}^{4 \delta} \mid d\left(x, \partial A_{\varepsilon}^{4 \delta}\right) \leq \sigma\right\}} K(\varepsilon x) Q\left(u_{\varepsilon}^{m}, v_{\varepsilon}^{m}\right) d x \\
& \geq 1-C \varepsilon^{(N-1)+3(p+1) /(p-1)}-C\left(\varepsilon / R_{0}\right)^{\gamma}>0
\end{aligned}
$$

for small $\varepsilon>0$ and for each $\sigma>0$. Then for each $\sigma>0$ there is a sequence $\left\{x_{m}\right\}_{m}$ in $A_{\varepsilon}^{4 \delta}$ such that $\lim _{m \rightarrow \infty} d\left(x_{m}, \partial A_{\varepsilon}^{4 \delta}\right)=0$ and $Q\left(u_{\varepsilon}^{m}\left(x_{m}\right), v_{\varepsilon}^{m}\left(x_{m}\right)\right)=$ 1. Since $A_{\varepsilon}^{4 \delta}$ is an compact subset of $\mathbb{R}^{N}$, we see that $\lim _{m \rightarrow \infty} x_{m}=x_{0} \in A_{\varepsilon}^{4 \delta}$, up to subsequence. This implies that $x_{0} \in \partial A_{\varepsilon}^{4 \delta}$ and $\lim _{m \rightarrow \infty}\left|x_{m}\right|=\left|x_{0}\right|=$ $r_{0}>0$ so that for each $\sigma>0$

$$
\liminf _{m \rightarrow \infty} \int_{D_{r_{0}}^{\sigma}} K(\varepsilon x) Q\left(u_{\varepsilon}^{m}, v_{\varepsilon}^{m}\right) d x>0
$$


where $D_{r_{0}}^{\sigma}$ is defined by $D_{r_{0}}^{\sigma} \equiv\left\{x \in \mathbb{R}^{N}\left|r_{0}-\sigma \leq\right| x \mid \leq r_{0}+\sigma\right\}$. To reach a contradiction of (18), we will prove the following statements:

$$
\int_{D_{r_{0}}^{\sigma}}\left[\left(\left(u_{\varepsilon}^{m}-1\right)_{+}\right)^{2}+\left(\left(v_{\varepsilon}^{m}-1\right)_{+}\right)^{2}\right] d x \leq C \sigma^{2 / N}\left\|\left(u_{\varepsilon}^{m}, v_{\varepsilon}^{m}\right)\right\|_{\varepsilon}^{2}
$$

for $m$ large and some positive constant $C$, independent of $\sigma$;

$$
\begin{aligned}
& \int_{D_{r_{0}}^{\sigma}}\left[\left|\nabla\left(u_{\varepsilon}^{m}-1\right)_{+}\right|^{2}+\left|\nabla\left(v_{\varepsilon}^{m}-1\right)_{+}\right|^{2}+V_{1}(\varepsilon x)\left(\left(u_{\varepsilon}^{m}-1\right)_{+}\right)^{2}\right. \\
& \left.+V_{2}(\varepsilon x)\left(\left(v_{\varepsilon}^{m}-1\right)_{+}\right)^{2}\right] d x \\
\leq & \left\|\left(u_{\varepsilon}^{m}, v_{\varepsilon}^{m}\right)\right\|_{\varepsilon}^{2}, \\
& \int_{D_{r_{0}}^{\sigma}} K(\varepsilon x) Q\left(\left(u_{\varepsilon}^{m}-1\right)_{+},\left(v_{\varepsilon}^{m}-1\right)_{+}\right) d x \leq C \sigma^{s(p+1) / N}
\end{aligned}
$$

for some $s \in(0,1)$ and $C>0, C$ independent of $\sigma$; and

$\int_{D_{r_{0}}^{\sigma}}\left[\left(u_{\varepsilon}^{m}\right)^{p+1}+\left(v_{\varepsilon}^{m}\right)^{p+1}\right] d x \leq C_{0} \int_{D_{r_{0}}^{\sigma}} K(\varepsilon x) Q\left(\left(u_{\varepsilon}^{m}-1\right)_{+},\left(v_{\varepsilon}^{m}-1\right)_{+}\right) d x+C_{0} \sigma$

for some positive constant $C_{0}$.

To prove the assertion (19) note that, by the Poincaré inequality, there is a positive constant $C_{1}$ so that

$$
\begin{aligned}
\int_{D_{r_{0}}^{\sigma}}\left(\left(u_{\varepsilon}^{m}-1\right)_{+}\right)^{2} d x & \leq\left(\left|D_{r_{0}}^{\sigma}\right| / \omega_{N}\right)^{2 / N} \int_{D_{r_{0}}^{\sigma}}\left|\nabla\left(u_{\varepsilon}^{m}-1\right)_{+}\right|^{2} d x \\
& \leq C_{1} \sigma^{2 / N} \int_{D_{r_{0}}^{\sigma}}\left|\nabla u_{\varepsilon}^{m}\right|^{2} d x \\
& \leq C_{1} \sigma^{2 / N}\left\|\left(u_{\varepsilon}^{m}, v_{\varepsilon}^{m}\right)\right\|_{\varepsilon}^{2} .
\end{aligned}
$$

Similarly $\int_{D_{r_{0}}^{\sigma}}\left(\left(v_{\varepsilon}^{m}-1\right)_{+}\right)^{2} d x \leq C_{2} \sigma^{2 / N}\left\|\left(u_{\varepsilon}^{m}, v_{\varepsilon}^{m}\right)\right\|_{\varepsilon}^{2}$ for some constant $C_{2}>0$ and the inequality (19) follows.

The verification of (20) is immediate.

For the statement (21), we use the interpolation inequality, Sobolev inequality, (19) and (20) to find

$$
\begin{aligned}
& \int_{D_{r_{0}}^{\sigma}}\left(\left(u_{\varepsilon}^{m}-1\right)_{+}\right)^{p+1} d x \\
\leq & C_{0}\left(\int_{D_{r_{0}}^{\sigma}}\left(\left(u_{\varepsilon}^{m}-1\right)_{+}\right)^{2} d x\right)^{s_{1}(p+1) / 2} \times\left(\int_{D_{r_{0}}^{\sigma}}\left|\nabla\left(u_{\varepsilon}^{m}-1\right)_{+}\right|^{2} d x\right)^{\left(1-s_{1}\right)(p+1) / 2} \\
\leq & C_{1}\left(\sigma^{2 / N}\left\|\left(u_{\varepsilon}^{m}, v_{\varepsilon}^{m}\right)\right\|_{\varepsilon}^{2}\right)^{s_{1}(p+1) / 2} \times\left(\left\|\left(u_{\varepsilon}^{m}, v_{\varepsilon}^{m}\right)\right\|_{\varepsilon}^{2}\right)^{\left(1-s_{1}\right)(p+1) / 2} \\
\leq & C_{2} \sigma^{s_{1}(p+1) / N}
\end{aligned}
$$


for $s_{1} \in(0,1)$ and for some constants $C_{0}, C_{1}, C_{2}>0$, independent of $\sigma$. Similarly, we get $\int_{D_{r_{0}}^{\sigma}}\left(\left(v_{\varepsilon}^{m}-1\right)_{+}\right)^{p+1} d x \leq C_{3} \sigma^{s_{2}(p+1) / N}$ for some constants $C_{3}>0$ and $s_{2} \in(0,1)$. Using this information and $\left(Q_{2}\right),(21)$ follows.

Finally, to obtain (22), we note that

$$
\begin{aligned}
\int_{D_{r_{0}}^{\sigma}}\left(u_{\varepsilon}^{m}\right)^{p+1} d x & \leq \int_{D_{r_{0}}^{\sigma} \cap\left\{u_{\varepsilon}^{m} \geq 1\right\}}\left(\left(u_{\varepsilon}^{m}-1\right)_{+}+1\right)^{p+1} d x+\left|D_{r_{0}}^{\sigma}\right| \\
& \leq 2^{p} \int_{D_{r_{0}}^{\sigma}}\left(\left(u_{\varepsilon}^{m}-1\right)_{+}\right)^{p+1} d x+\left(2^{p}+1\right)\left|D_{r_{0}}^{\sigma}\right|
\end{aligned}
$$

Also, $\int_{D_{r_{0}}^{\sigma}}\left(v_{\varepsilon}^{m}\right)^{p+1} d x \leq 2^{p} \int_{D_{r_{0}}^{\sigma}}\left(\left(v_{\varepsilon}^{m}-1\right)_{+}\right)^{p+1} d x+\left(2^{p}+1\right)\left|D_{r_{0}}^{\sigma}\right|$. Therefore

$$
\begin{aligned}
& \int_{D_{r_{0}}^{\sigma}}\left[\left(u_{\varepsilon}^{m}\right)^{p+1}+\left(v_{\varepsilon}^{m}\right)^{p+1}\right] d x \\
\leq & 2^{p} \int_{D_{r_{0}}^{\sigma}}\left[\left(\left(u_{\varepsilon}^{m}-1\right)_{+}\right)^{p+1}+\left(\left(v_{\varepsilon}^{m}-1\right)_{+}\right)^{p+1}\right] d x+2\left(2^{p}+1\right)\left|D_{r_{0}}^{\sigma}\right| .
\end{aligned}
$$

Using (23), $\left(Q_{2}\right)$ and the fact that $\left|D_{r_{0}}^{\sigma}\right| \leq C \sigma$ for all smal $\sigma>0$ and for some positive constant $C$, we obtain (22). From $\left(Q_{2}\right),(21)$ and $(22)$ it follows that

$$
\begin{aligned}
\int_{D_{r_{0}}^{\sigma}} K(\varepsilon x) Q\left(u_{\varepsilon}^{m}, v_{\varepsilon}^{m}\right) d x & \leq C_{0} \eta_{2} \int_{D_{r_{0}}^{\sigma}}\left[\left(u_{\varepsilon}^{m}\right)^{p+1}+\left(v_{\varepsilon}^{m}\right)^{p+1}\right] d x \\
& \leq C_{1} \int_{D_{r_{0}}^{\sigma}} K(\varepsilon x) Q\left(\left(u_{\varepsilon}^{m}-1\right)_{+},\left(v_{\varepsilon}^{m}-1\right)_{+}\right) d x+C_{1} \sigma \\
& \leq C\left(\sigma^{s(p+1) / N}+\sigma\right)
\end{aligned}
$$

for some $s \in(0,1)$ and for some constants $C_{0}, C_{1}, C>0$, independent of $\sigma$ and $m$. Therefore,

$$
\liminf _{m \rightarrow \infty} \int_{D_{r_{0}}^{\sigma}} K(\varepsilon x) Q\left(u_{\varepsilon}^{m}, v_{\varepsilon}^{m}\right) d x \leq C\left(\sigma^{s(p+1) / N}+\sigma\right)
$$

for all $\sigma>0$ small. But this contradicts (18), given the arbitrariness of $\sigma>0$. Thus, we conclude that $\left\{\alpha_{\varepsilon}^{m}\right\}_{m}$ is bounded. This implies that $\lim _{m \rightarrow \infty} \alpha_{\varepsilon}^{m}=$ $\alpha_{\varepsilon} \geq 0$, up to subsequence. Using (12) and the fact that

$$
0 \leq \int_{\mathbb{R}^{N}} \chi_{\varepsilon}(x) Q\left(u_{\varepsilon}^{m}, v_{\varepsilon}^{m}\right) d x \leq 1
$$

for all $m \in \mathbb{N}$, we get $\lim _{m \rightarrow \infty} \beta_{\varepsilon}^{m}=\beta_{\varepsilon} \leq 0$. Since $\left(u_{\varepsilon}^{m}, v_{\varepsilon}^{m}\right)$ is solution of $\left(S_{\alpha_{\varepsilon}^{m}, \beta_{\varepsilon}^{m}}\right)$ we have that

$$
\begin{aligned}
& \int_{\mathbb{R}^{N}}\left[\nabla u_{\varepsilon}^{m} \cdot \nabla \varphi+\nabla v_{\varepsilon}^{m} \cdot \nabla \psi+V_{1}(\varepsilon x) u_{\varepsilon}^{m} \varphi+V_{2}(\varepsilon x) v_{\varepsilon}^{m} \psi\right] d x \\
= & \alpha_{\varepsilon}^{m} \int_{\mathbb{R}^{N}} K(\varepsilon x)\left[\varphi Q_{u}\left(u_{\varepsilon}^{m}, v_{\varepsilon}^{m}\right)+\psi Q_{v}\left(u_{\varepsilon}^{m}, v_{\varepsilon}^{m}\right)\right] d x
\end{aligned}
$$




$$
+\beta_{\varepsilon}^{m} \int_{\mathbb{R}^{N}} \chi_{\varepsilon}\left[\varphi Q_{u}\left(u_{\varepsilon}^{m}, v_{\varepsilon}^{m}\right)+\psi Q_{v}\left(u_{\varepsilon}^{m}, v_{\varepsilon}^{m}\right)\right] d x
$$

for any $\varphi, \psi \in C_{0, \text { rad }}^{\infty}\left(\mathbb{R}^{N}\right)$. Finally, taking the limit in (24) as $m \rightarrow \infty$, we see that

$$
\begin{aligned}
& \left.\int_{\mathbb{R}^{N}}\left[\nabla \bar{u}_{\varepsilon} \cdot \nabla \varphi+\nabla \bar{v}_{\varepsilon} \cdot \nabla \psi+V_{1}(\varepsilon x) \varphi \bar{u}_{\varepsilon}+V_{2}(\varepsilon x) \psi \bar{v}_{\varepsilon}\right)\right] d x \\
= & \alpha_{\varepsilon} \int_{\mathbb{R}^{N}} K(\varepsilon x)\left[\varphi Q_{u}\left(\bar{u}_{\varepsilon}, \bar{v}_{\varepsilon}\right)+\psi Q_{v}\left(\bar{u}_{\varepsilon}, \bar{v}_{\varepsilon}\right)\right] d x \\
& +\beta_{\varepsilon} \int_{\mathbb{R}^{N}} \chi_{\varepsilon}\left[\varphi Q_{u}\left(\bar{u}_{\varepsilon}, \bar{v}_{\varepsilon}\right)+\psi Q_{v}\left(\bar{u}_{\varepsilon}, \bar{v}_{\varepsilon}\right)\right] d x
\end{aligned}
$$

for any $\varphi, \psi \in C_{0, \text { rad }}^{\infty}\left(\mathbb{R}^{N}\right)$. Therefore, $\left(\bar{u}_{\varepsilon}, \bar{v}_{\varepsilon}\right)$ satisfies $\left(S_{\alpha_{\varepsilon}, \beta_{\varepsilon}}\right)$. From (7), $\beta_{\varepsilon} \leq 0$, the homogeneity of $Q$ and the fact that $\left(\bar{u}_{\varepsilon}, \bar{v}_{\varepsilon}\right)$ is solution of $\left(S_{\alpha_{\varepsilon}, \beta_{\varepsilon}}\right)$, we conclude that $\left\|\left(\bar{u}_{\varepsilon}, \bar{v}_{\varepsilon}\right)\right\|_{\varepsilon}^{2} \leq(p+1) \alpha_{\varepsilon}$ and therefore $\alpha_{\varepsilon}>0$. This completes the proof of lemma.

Claim: For $\varepsilon$ small,

$$
\int_{\mathbb{R}^{N}} \chi_{\varepsilon} Q\left(\bar{u}_{\varepsilon}, \bar{v}_{\varepsilon}\right) d x<1
$$

This claim is one of the crucial setps of our work. We will postpone its proof for while. If this is the case, for any $\varphi, \psi \in C_{0, \text { rad }}^{\infty}\left(\mathbb{R}^{N}\right)$, we define

$$
\varphi_{s} \equiv\left(\bar{u}_{\varepsilon}+s \varphi\right)\left(\int_{\mathbb{R}^{N}} K(\varepsilon x) Q\left(\bar{u}_{\varepsilon}+s \varphi, \bar{v}_{\varepsilon}+s \psi\right) d x\right)^{-1 /(p+1)}
$$

and

$$
\psi_{s} \equiv\left(\bar{v}_{\varepsilon}+s \psi\right)\left(\int_{\mathbb{R}^{N}} K(\varepsilon x) Q\left(\bar{u}_{\varepsilon}+s \varphi, \bar{v}_{\varepsilon}+s \psi\right) d x\right)^{-1 /(p+1)} .
$$

From (7) we conclude that $\left(\varphi_{0}, \psi_{0}\right)=\left(\bar{u}_{\varepsilon}, \bar{v}_{\varepsilon}\right)$. Since $Q$ is homogeneous of degree $p+1$, we obtain $\int_{\mathbb{R}^{N}} K(\varepsilon x) Q\left(\varphi_{s}, \psi_{s}\right) d x=1$. Also, by $(25), \int_{\mathbb{R}^{N}} \chi_{\varepsilon} Q\left(\varphi_{s}, \psi_{s}\right) d x<$ 1 for small $|s|$.

Thus,

$$
\begin{aligned}
0= & \left.\frac{d}{d s}\left\|\left(\varphi_{s}, \psi_{s}\right)\right\|_{\varepsilon}^{2}\right|_{s=0} \\
= & -2 M_{\varepsilon} /(p+1) \int_{\mathbb{R}^{N}} K(\varepsilon x)\left[\varphi Q_{u}\left(\bar{u}_{\varepsilon}, \bar{v}_{\varepsilon}\right)+\psi Q_{v}\left(\bar{u}_{\varepsilon}, \bar{v}_{\varepsilon}\right)\right] d x \\
& +2 \int_{\mathbb{R}^{N}}\left[\nabla \bar{u}_{\varepsilon} \cdot \nabla \varphi+\nabla \bar{v}_{\varepsilon} \cdot \nabla \psi+V_{1}(\varepsilon x) \bar{u}_{\varepsilon} \varphi+V_{2}(\varepsilon x) \bar{v}_{\varepsilon} \psi\right] d x .
\end{aligned}
$$

This implies that $\left(\bar{u}_{\varepsilon}, \bar{v}_{\varepsilon}\right)$ satisfies the system $\left(S_{M_{\varepsilon} /(p+1), 0}\right)$. Then, as the functions $Q_{u}$ and $Q_{v}$ are homogeneous of degree $p$, we deduce that $\left(\tilde{u}_{\varepsilon}, \tilde{v}_{\varepsilon}\right)$, where $\tilde{u}_{\varepsilon}=\left(M_{\varepsilon} /(p+1)\right)^{1 /(p-1)} \bar{u}_{\varepsilon}$ and $\tilde{v}_{\varepsilon}=\left(M_{\varepsilon} /(p+1)\right)^{1 /(p-1)} \bar{v}_{\varepsilon}$, is a solution of $(\tilde{S})$.

Lemma 4. $\lim _{\varepsilon \rightarrow 0} \varepsilon^{(N-1)(p-1) /(p+1)} \alpha_{\varepsilon}=0$. 
Proof. Assume by contradiction, taking a subsequence if necessary, that

$$
\lim _{\varepsilon \rightarrow 0} \varepsilon^{(N-1)(p-1) /(p+1)} \alpha_{\varepsilon}=\alpha \in(0, \infty] .
$$

For any $\sigma>0$, we choose $\phi_{\sigma} \in C_{0}^{\infty}\left(\operatorname{int}\left(A_{\varepsilon}^{4 \delta}\right)\right)$ satisfying $0 \leq \phi_{\sigma} \leq 1, \phi_{\sigma}(x)=1$ for $d\left(x, \partial A_{\varepsilon}^{4 \delta}\right) \geq \sigma$, and $\left|\nabla \phi_{\sigma}\right| \leq 2 / \sigma$. Using $\phi_{\sigma}\left(\bar{u}_{\varepsilon}, \bar{v}_{\varepsilon}\right)$ as test function in the system $\left(S_{\alpha_{\varepsilon}, \beta_{\varepsilon}}\right)$ and the fact that $\chi_{\varepsilon} \phi_{\sigma} \equiv 0$, we have

$$
\begin{aligned}
& \int_{\mathbb{R}^{N}}\left[\left|\nabla \bar{u}_{\varepsilon}\right|^{2} \phi_{\sigma}+\nabla \bar{u}_{\varepsilon} \cdot \nabla \phi_{\sigma} \bar{u}_{\varepsilon}+\left|\nabla \bar{v}_{\varepsilon}\right|^{2} \phi_{\sigma}+\nabla \bar{v}_{\varepsilon} \cdot \nabla \phi_{\sigma} \bar{v}_{\varepsilon}\right. \\
& \left.+\phi_{\sigma}\left(V_{1}(\varepsilon x)\left(\bar{u}_{\varepsilon}\right)^{2}+V_{2}(\varepsilon x)\left(\bar{v}_{\varepsilon}\right)^{2}\right)\right] d x \\
= & (p+1) \alpha_{\varepsilon} \int_{\mathbb{R}^{N}} K(\varepsilon x) Q\left(\bar{u}_{\varepsilon}, \bar{v}_{\varepsilon}\right) \phi_{\sigma} d x .
\end{aligned}
$$

From $\inf _{x \in \operatorname{supp}\left(\left|\nabla \phi_{\sigma}\right|\right)} V(\varepsilon x)>0$ and the properties of $\phi_{\sigma}$, we obtain

$$
\begin{aligned}
& \int_{\mathbb{R}^{N}}\left[\left|\nabla \bar{u}_{\varepsilon}\right|^{2} \phi_{\sigma}+\nabla \bar{u}_{\varepsilon} \cdot \nabla \phi_{\sigma} \bar{u}_{\varepsilon}+\left|\nabla \bar{v}_{\varepsilon}\right|^{2} \phi_{\sigma}+\nabla \bar{v}_{\varepsilon} \cdot \nabla \phi_{\sigma} \bar{v}_{\varepsilon}\right. \\
& \left.+\phi_{\sigma}\left(V_{1}(\varepsilon x)\left(\bar{u}_{\varepsilon}\right)^{2}+V_{2}(\varepsilon x)\left(\bar{v}_{\varepsilon}\right)^{2}\right)\right] d x \\
\leq & C\left\|\left(\bar{u}_{\varepsilon}, \bar{v}_{\varepsilon}\right)\right\|_{\varepsilon}^{2}
\end{aligned}
$$

for some $C>0$, independent of $\varepsilon>0$. From (26) and (27) it follows that $\int_{\mathbb{R}^{N}} K(\varepsilon x) Q\left(\bar{u}_{\varepsilon}, \bar{v}_{\varepsilon}\right) \phi_{\sigma} d x \leq C\left\|\left(\bar{u}_{\varepsilon}, \bar{v}_{\varepsilon}\right)\right\|_{\varepsilon}^{2} / \alpha_{\varepsilon}$ for some positive constant $C$, independent of $\varepsilon>0$. By Lemma 2, for each $\sigma>0$,

$$
\lim _{\varepsilon \rightarrow 0} \int_{\mathbb{R}^{N}} K(\varepsilon x) Q\left(\bar{u}_{\varepsilon}, \bar{v}_{\varepsilon}\right) \phi_{\sigma} d x=0 .
$$

Then

$$
\lim _{\varepsilon \rightarrow 0} \int_{\left\{x \in A_{\varepsilon}^{4 \delta} \mid d\left(x, \partial A_{\varepsilon}^{4 \delta}\right) \geq \sigma\right\}} K(\varepsilon x) Q\left(\bar{u}_{\varepsilon}, \bar{v}_{\varepsilon}\right) d x=0 .
$$

From (8) and the definition of $\chi_{\varepsilon}$, we get

$$
\int_{\mathbb{R}^{N} \backslash B\left(0, R_{0} / \varepsilon\right)} K(\varepsilon x) Q\left(\bar{u}_{\varepsilon}, \bar{v}_{\varepsilon}\right) d x \leq C\left(\varepsilon / R_{0}\right)^{\gamma}
$$

and

$$
\int_{B\left(0, R_{0} / \varepsilon\right) \backslash A_{\varepsilon}^{4 \delta}} K(\varepsilon x) Q\left(\bar{u}_{\varepsilon}, \bar{v}_{\varepsilon}\right) d x \leq C \varepsilon^{(N-1)+3(p+1) /(p-1)}
$$

for some $C>0$. From (7), (28), (29) and (30), we see that for each $\sigma>0$,

$$
\liminf _{\varepsilon \rightarrow 0} \int_{\left\{x \in A_{\varepsilon}^{4 \delta} \mid d\left(x, \partial A_{\varepsilon}^{4 \delta}\right) \leq \sigma\right\}} K(\varepsilon x) Q\left(\bar{u}_{\varepsilon}, \bar{v}_{\varepsilon}\right) d x>0 .
$$

From (31), for every $\sigma>0$, there exists a sequence $\left\{x_{m}\right\}_{m}$ in $A_{\varepsilon}^{4 \delta}$ such that $\lim _{m \rightarrow \infty} d\left(x_{m}, \partial A_{\varepsilon}^{4 \delta}\right)=0$. Therefore, there exists some $x_{0} \in \partial A^{4 \delta}$, with 
$\lim _{m \rightarrow \infty} x_{m}=\frac{x_{0}}{\varepsilon}$, and $\omega>0$ such that for any $\sigma>0$,

$$
\liminf _{\varepsilon \rightarrow 0} \int_{\left\{x \in \mathbb{R}^{N}\right.} K(\varepsilon x) Q\left(\bar{u}_{\varepsilon}, \bar{v}_{\varepsilon}\right) d x \geq \omega,
$$

otherwise we would have $\lim _{\varepsilon \rightarrow 0} \int_{\mathbb{R}^{N}} K(\varepsilon x) Q\left(\bar{u}_{\varepsilon}, \bar{v}_{\varepsilon}\right) d x=0$. But this is impossible because it contradicts $(7)$.

We fix $\sigma>0$ and choose a radially symmetric function $\psi_{\sigma} \in C_{0}^{\infty}$ so that

$$
\psi_{\sigma}(x)= \begin{cases}0 & \text { if } \quad|| x|-| x_{0}|/ \varepsilon| \geq 2 \sigma \\ 1 & \text { if } \quad|| x|-| x_{0}|/ \varepsilon| \leq \sigma\end{cases}
$$

$0 \leq \psi_{\sigma} \leq 1$ and $\left|\nabla \psi_{\sigma}\right| \leq 3 / \sigma$. From (32) it follows that

$$
\liminf _{\varepsilon \rightarrow 0} \int_{\mathbb{R}^{N}} K(\varepsilon x) Q\left(\psi_{\sigma} \bar{u}_{\varepsilon}, \psi_{\sigma} \bar{v}_{\varepsilon}\right) d x \geq \omega .
$$

Now, we claim that

$$
\lim _{\varepsilon \rightarrow 0} \varepsilon^{(N-1)(p-1) /(p+1)}\left\|\left(\psi_{\sigma} \bar{u}_{\varepsilon}, \psi_{\sigma} \bar{v}_{\varepsilon}\right)\right\|_{\varepsilon}^{2}=0 .
$$

Indeed, by the Cauchy-Schwarz inequality, the boundedness of the gradient of $\psi_{\sigma}$, and by the fact that $\alpha_{0}=\inf _{x \in \operatorname{supp}\left(\psi_{\sigma}\right)} V(\varepsilon x)>0$, we have

$$
\begin{aligned}
& \left\|\left(\psi_{\sigma} \bar{u}_{\varepsilon}, \psi_{\sigma} \bar{v}_{\varepsilon}\right)\right\|_{\varepsilon}^{2} \\
\leq & C_{1} \int_{\operatorname{supp}\left(\psi_{\sigma}\right)}\left[\left|\nabla \bar{u}_{\varepsilon}\right|^{2}+\left|\nabla \bar{v}_{\varepsilon}\right|^{2}+\left(\bar{u}_{\varepsilon}\right)^{2}+\left(\bar{v}_{\varepsilon}\right)^{2}+V_{1}(\varepsilon x)\left(\bar{u}_{\varepsilon}\right)^{2}\right. \\
& \left.+V_{2}(\varepsilon x)\left(\bar{v}_{\varepsilon}\right)^{2}\right] d x \\
\leq & C_{1} \int_{\operatorname{supp}\left(\psi_{\sigma}\right)}\left[\left|\nabla \bar{u}_{\varepsilon}\right|^{2}+\left|\nabla \bar{v}_{\varepsilon}\right|^{2}+\frac{1}{\alpha_{0}} V_{1}(\varepsilon x)\left(\bar{u}_{\varepsilon}\right)^{2}+\frac{1}{\alpha_{0}} V_{2}(\varepsilon x)\left(\bar{v}_{\varepsilon}\right)^{2}\right. \\
& \left.+V_{1}(\varepsilon x)\left(\bar{u}_{\varepsilon}\right)^{2}+V_{2}(\varepsilon x)\left(\bar{v}_{\varepsilon}\right)^{2}\right] d x \\
\leq & C_{2}\left\|\left(\bar{u}_{\varepsilon}, \bar{v}_{\varepsilon}\right)\right\|_{\varepsilon}^{2}=C_{2} M_{\varepsilon}
\end{aligned}
$$

for some positive constants $C_{1}$ and $C_{2}$, independent of $\varepsilon>0$. By Lemma 2, (34) follows.

On other hand, putting $D_{\varepsilon} \equiv\left\{x \in \mathbb{R}^{N}|| x_{0}|/ \varepsilon-2 \sigma \leq| x|\leq| x_{0} \mid / \varepsilon+2 \sigma\right\}$, we see that

$$
\begin{aligned}
& \liminf _{\varepsilon \rightarrow 0} \varepsilon^{(N-1)(p-1) /(p+1)}\left\|\left(\psi_{\sigma} \bar{u}_{\varepsilon}, \psi_{\sigma} \bar{v}_{\varepsilon}\right)\right\|_{\varepsilon}^{2} \\
\geq & \liminf _{\varepsilon \rightarrow 0}\left(\left[\int_{\mathbb{R}^{N}} K(\varepsilon x) Q\left(\psi_{\sigma} \bar{u}_{\varepsilon}, \psi_{\sigma} \bar{v}_{\varepsilon}\right) d x\right]^{2 /(p+1)}\right) \\
& \times \liminf _{\varepsilon \rightarrow 0}\left(\varepsilon^{(N-1)(p-1) /(p+1)} \inf _{u, v \in C_{0}^{1}\left(D_{\varepsilon}\right)} \frac{\|(u, v)\|_{\varepsilon}^{2}}{\left[\int_{\mathbb{R}^{N}} K(\varepsilon x) Q(u, v) d x\right]^{2 /(p+1)}}\right) \\
\geq & C \omega^{2 /(p+1)} \liminf _{\varepsilon \rightarrow 0}\left(\left(\left|x_{0}\right|-2 \sigma \varepsilon\right)^{N-1}\left(\left|x_{0}\right|+2 \sigma \varepsilon\right)^{-2(N-1) /(p+1)}\right) J_{\sigma} \\
(35)= & C \omega^{2 /(p+1)}\left|x_{0}\right|^{(N-1)(p-1) /(p+1)} J_{\sigma}>0
\end{aligned}
$$


for some $C>0$, where

$$
J_{\sigma} \equiv \inf _{g_{0}, g_{1} \in C_{0}^{1}(-2 \sigma, 2 \sigma)} \frac{\int_{-2 \sigma}^{2 \sigma}\left[\left(g_{0}^{\prime}(s)\right)^{2}+\left(g_{1}^{\prime}(s)\right)^{2}+\alpha_{0}\left(\left(g_{0}(s)\right)^{2}+\left(g_{1}(s)\right)^{2}\right)\right] d s}{\left[\int_{-2 \sigma}^{2 \sigma}\left|g_{0}(s)\right|^{p+1} d s\right]^{2 /(p+1)}+\left[\int_{-2 \sigma}^{2 \sigma}\left|g_{1}(s)\right|^{p+1} d s\right]^{2 /(p+1)}} .
$$

From (34) and (35) we have a contradiction. So, to conclude the proof of the lemma, we will have a verification for (35).

Using $\left(Q_{2}\right)$, change of variables and setting $g_{0}\left(s+\left|x_{0}\right| / \varepsilon\right)=\bar{g}_{0}(s), g_{1}(s+$ $\left.\left|x_{0}\right| / \varepsilon\right)=\bar{g}_{1}(s)$, we deduce that

$$
\begin{aligned}
& \frac{\|(u, v)\|_{\varepsilon}^{2}}{\left[\int_{\mathbb{R}^{N}} K(\varepsilon x) Q(u, v) d x\right]^{2 /(p+1)}} \\
\geq & C \varepsilon^{-(N-1)(p-1) /(p+1)}\left(\left|x_{0}\right|-2 \sigma \varepsilon\right)^{N-1}\left(\left|x_{0}\right|+2 \sigma \varepsilon\right)^{-2(N-1) /(p+1)} \\
& \times \frac{\int_{-2 \sigma}^{2 \sigma}\left[\left(\bar{g}_{0}^{\prime}(s)\right)^{2}+\left(\bar{g}_{1}^{\prime}(s)\right)^{2}+\alpha_{0}\left(\left(\bar{g}_{0}(s)\right)^{2}+\left(\bar{g}_{1}(s)\right)^{2}\right)\right] d s}{\left(\int_{-2 \sigma}^{2 \sigma}\left|\bar{g}_{0}(s)\right|^{p+1} d s\right)^{2 /(p+1)}+\left(\int_{-2 \sigma}^{2 \sigma}\left|\bar{g}_{1}(s)\right|^{p+1} d s\right)^{2 /(p+1)}} \\
\geq & C \varepsilon^{-(N-1)(p-1) /(p+1)}\left(\left|x_{0}\right|-2 \sigma \varepsilon\right)^{N-1}\left(\left|x_{0}\right|+2 \sigma \varepsilon\right)^{-2(N-1) /(p+1)} J_{\sigma}
\end{aligned}
$$

for some positive constant $C$. Then

$$
\begin{aligned}
& \varepsilon^{(N-1)(p-1) /(p+1)} \inf _{u, v \in C_{0}^{1}\left(D_{\varepsilon}\right)} \frac{\|(u, v)\|_{\varepsilon}^{2}}{\left[\int_{\mathbb{R}^{N}} K(\varepsilon x) Q(u, v) d x\right]^{2 /(p+1)}} \\
\geq & C\left(\left|x_{0}\right|-2 \sigma \varepsilon\right)^{N-1}\left(\left|x_{0}\right|+2 \sigma \varepsilon\right)^{-2(N-1) /(p+1)} J_{\sigma} .
\end{aligned}
$$

Combining (33) and (36) we obtain (35). The proof of the lemma is complete.

Lemma 5. If $\left(\bar{u}_{\varepsilon}, \bar{v}_{\varepsilon}\right)$ and $\alpha_{\varepsilon}$ are as above, then

$$
\lim _{\varepsilon \rightarrow 0}\left\|\left(\alpha_{\varepsilon}\right)^{1 /(p-1)} \bar{u}_{\varepsilon}\right\|_{L^{\infty}\left(\mathbb{R}^{N}\right)}=\lim _{\varepsilon \rightarrow 0}\left\|\left(\alpha_{\varepsilon}\right)^{1 /(p-1)} \bar{v}_{\varepsilon}\right\|_{L^{\infty}\left(\mathbb{R}^{N}\right)}=0 .
$$

Proof. Let $w_{\varepsilon}=\left(\alpha_{\varepsilon}\right)^{1 /(p-1)}\left(\bar{u}_{\varepsilon}+\bar{v}_{\varepsilon}\right)$. By $\left(Q_{1}\right),\left(Q_{5}\right)$ and the fact that $\left(\bar{u}_{\varepsilon}, \bar{v}_{\varepsilon}\right)$ is solution $\left(S_{\alpha_{\varepsilon}, \beta_{\varepsilon}}\right)$ it follows that

$$
-\Delta w_{\varepsilon}+V(\varepsilon x) w_{\varepsilon} \leq C K(\varepsilon x)\left(w_{\varepsilon}\right)^{p} \text { in } \mathbb{R}^{N}
$$

for some positive constant $C$.

Now, we claim that

$$
\left.\left.\lim _{\varepsilon \rightarrow 0}|| w_{\varepsilon} \|_{L^{\infty}\left(\left\{x \in \mathbb{R}^{N}\right.\right.}|| y|/ \varepsilon-1 \leq| x|\leq| y \mid / \varepsilon+1\right\}\right)=0
$$

for all $y \in \mathbb{R}^{N} \backslash\{0\}$ and

$$
\lim _{\varepsilon \rightarrow 0}\left\|w_{\varepsilon}\right\|_{L^{\infty}\left(B\left(0, r_{0} / \varepsilon\right)\right)}=0
$$

for some constant $r_{0}>0$. 
Proof of (38): First of all we will show that

$$
\lim _{\varepsilon \rightarrow 0} \int_{B(y / \varepsilon, 2)}\left(\alpha_{\varepsilon}\right)^{(p+1) /(p-1)} K(\varepsilon x) Q\left(\bar{u}_{\varepsilon}, \bar{v}_{\varepsilon}\right) d x=0,
$$

all $y \in \mathbb{R}^{N} \backslash\{0\}$. Suppose that

$$
\liminf _{\varepsilon \rightarrow 0} \int_{B(y / \varepsilon, 2)}\left(\alpha_{\varepsilon}\right)^{(p+1) /(p-1)} K(\varepsilon x) Q\left(\bar{u}_{\varepsilon}, \bar{v}_{\varepsilon}\right) d x>0
$$

for some $y \in \mathbb{R}^{N} \backslash\{0\}$. As $K, \bar{u}_{\varepsilon}$ and $\bar{v}_{\varepsilon}$ are radially symmetric functions, it follows that

$$
\begin{aligned}
& (\varepsilon /|y|)^{N-1} \int_{\left\{x \in \mathbb{R}^{N}|| y|/ \varepsilon-2 \leq| x|\leq| y \mid / \varepsilon+2\right\}}\left(\alpha_{\varepsilon}\right)^{(p+1) /(p-1)} K(\varepsilon x) Q\left(\bar{u}_{\varepsilon}, \bar{v}_{\varepsilon}\right) d x \\
\geq & C \int_{B(y / \varepsilon, 2)}\left(\alpha_{\varepsilon}\right)^{(p+1) /(p-1)} K(\varepsilon x) Q\left(\bar{u}_{\varepsilon}, \bar{v}_{\varepsilon}\right) d x .
\end{aligned}
$$

This implies that

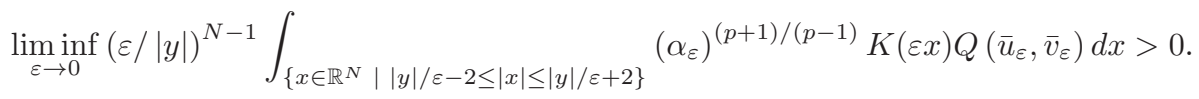

In view of (7) and Lemma 4 , we have a contradiction. Similarly,

$$
\limsup _{\varepsilon \rightarrow 0} \int_{B(y / \varepsilon, 2)}\left(\alpha_{\varepsilon}\right)^{(p+1) /(p-1)} K(\varepsilon x) Q\left(\bar{u}_{\varepsilon}, \bar{v}_{\varepsilon}\right) d x=0, \forall y \in \mathbb{R}^{N} \backslash\{0\}
$$

and the proof of (40) is complete.

Note that

$$
\int_{B(y / \varepsilon, 2)}\left(w_{\varepsilon}\right)^{p+1} d x \leq C \int_{B(y / \varepsilon, 2)}\left(\alpha_{\varepsilon}\right)^{(p+1) /(p-1)} K(\varepsilon x) Q\left(\bar{u}_{\varepsilon}, \bar{v}_{\varepsilon}\right) d x
$$

for some $C>0$. From this and (40) we see that

$$
\lim _{\varepsilon \rightarrow 0} \int_{B(y / \varepsilon, 2)}\left(w_{\varepsilon}\right)^{p+1} d x=0
$$

for all $y \in \mathbb{R}^{N} \backslash\{0\}$. Now we fix $\varepsilon>0$. Using (37) and the fact that $w_{\varepsilon}$ is radially symmetric we deduce, by the Moser iteration argument (see Theorem 9.20 in [26]), that

$$
\left.\left.\left\|w_{\varepsilon}\right\|_{L^{\infty}\left(\left\{x \in \mathbb{R}^{N}\right.\right.}|| y|/ \varepsilon-1 \leq| x|\leq| y \mid / \varepsilon+1\right\}\right) \leq C\left(\int_{B(y / \varepsilon, 2)}\left(w_{\varepsilon}\right)^{p+1} d x\right)^{1 /(p+1)}
$$

for some positive constant $C$, independent of $\varepsilon>0$. Using this and (41) we obtain (38).

Proof of (39): From (8), the definition of $\chi_{\varepsilon}$ and the fact that $0 \notin A^{4 \delta}$, it follows that there is a constant $r_{0}>0$ such that

$$
\int_{B\left(0,2 r_{0} / \varepsilon\right)} K(\varepsilon x) Q\left(\bar{u}_{\varepsilon}, \bar{v}_{\varepsilon}\right) d x \leq C \varepsilon^{(N-1)+3(p+1) /(p-1)}
$$


for small $\varepsilon>0$ and for some $C>0$. By (42) and Lemma 4, we have

$$
\int_{B\left(0,2 r_{0} / \varepsilon\right)}\left(\alpha_{\varepsilon}\right)^{(p+1) /(p-1)} K(\varepsilon x) Q\left(\bar{u}_{\varepsilon}, \bar{v}_{\varepsilon}\right) d x \leq C \varepsilon^{3(p+1) /(p-1)}
$$

for small $\varepsilon>0$ and for some $C>0$. As $\left(Q_{2}\right)$ and (43) are satisfied, we see that

$$
\int_{B\left(0,2 r_{0} / \varepsilon\right)}\left(w_{\varepsilon}\right)^{p+1} d x \leq C \varepsilon^{3(p+1) /(p-1)}
$$

for small $\varepsilon>0$ and for some $C>0$. Then using Theorem 9.20 in [26] and (44) we conclude that

$$
\left\|w_{\varepsilon}\right\|_{L^{\infty}\left(B\left(0, r_{0} / \varepsilon\right)\right)} \leq C \varepsilon^{N /(p+1)} \varepsilon^{3 /(p-1)}
$$

for some positive constant $C$, independent of $\varepsilon>0$. This shows (39). From (38) and (39) the lemma follows.

In the next lemma, we also will use the arguments developed by Byeon and Wang in [18] adapted to our case.

Lemma 6. $\liminf _{\varepsilon \rightarrow 0} \varepsilon^{-2} \alpha_{\varepsilon}>0$.

Proof. On the contrary, we assume for a subsequence, still denoted by $\varepsilon$, that $\varepsilon^{-2} \alpha_{\varepsilon} \rightarrow 0$ as $\varepsilon \rightarrow 0$. Let $\phi$ be a cut-off function such that $\phi(x)=1$ for $x \in$ $A_{\varepsilon}^{4 \delta}, \phi(x)=0$ for $x \notin A_{\varepsilon}^{5 \delta}, 0 \leq \phi \leq 1$ and $|\nabla \phi| \leq c \varepsilon, c>0$. Then, it follows that

$$
\lim _{\varepsilon \rightarrow 0} \int_{\mathbb{R}^{N}}\left[\left|\nabla\left(\phi \bar{u}_{\varepsilon}\right)\right|^{2}+\left|\nabla\left(\phi \bar{v}_{\varepsilon}\right)\right|^{2}\right] d x=0 .
$$

In fact, since $V(\varepsilon x) \geq \delta_{0}>0$ for all $x \in \operatorname{supp}(|\nabla \phi|)$, we see that

$$
\begin{aligned}
& \int_{\mathbb{R}^{N}}\left[\left|\nabla\left(\phi \bar{u}_{\varepsilon}\right)\right|^{2}+\left|\nabla\left(\phi \bar{v}_{\varepsilon}\right)\right|^{2}\right] d x \\
\leq & 2(c \varepsilon)^{2} \delta_{0}^{-1} \int_{\operatorname{supp}(|\nabla \phi|)}\left[V_{1}(\varepsilon x)\left(\bar{u}_{\varepsilon}\right)^{2}+V_{2}(\varepsilon x)\left(\bar{v}_{\varepsilon}\right)^{2}\right] d x \\
& +2 \int_{\mathbb{R}^{N}}\left[\left|\nabla \bar{u}_{\varepsilon}\right|^{2}+\left|\nabla \bar{v}_{\varepsilon}\right|^{2}\right] d x \\
\leq & C \int_{\mathbb{R}^{N}}\left[\left|\nabla \bar{u}_{\varepsilon}\right|^{2}+\left|\nabla \bar{v}_{\varepsilon}\right|^{2}+V_{1}(\varepsilon x)\left(\bar{u}_{\varepsilon}\right)^{2}+V_{2}(\varepsilon x)\left(\bar{v}_{\varepsilon}\right)^{2}\right] d x=C\left\|\left(\bar{u}_{\varepsilon}, \bar{v}_{\varepsilon}\right)\right\|_{\varepsilon}^{2}
\end{aligned}
$$

for some positive constant $C$, independent of small $\varepsilon>0$. This and Lemma 3 imply

$$
\int_{\mathbb{R}^{N}}\left[\left|\nabla\left(\phi \bar{u}_{\varepsilon}\right)\right|^{2}+\left|\nabla\left(\phi \bar{v}_{\varepsilon}\right)\right|^{2}\right] d x \leq C_{1} \alpha_{\varepsilon} \leq C_{1} \varepsilon^{-2} \alpha_{\varepsilon}
$$

for some $C_{1}>0$ independent of small $\varepsilon>0$. Hence, we get (45).

Now, using change of variables, $\left(Q_{2}\right)$, the Hölder inequality and Sobolev imbedding results, we see that

$$
\int_{A_{\varepsilon}^{4 \delta}} K(\varepsilon x) Q\left(\bar{u}_{\varepsilon}(x), \bar{v}_{\varepsilon}(x)\right) d x
$$




$$
\begin{aligned}
\leq & C\left|A^{4 \delta}\right|^{\left(2^{*}-(p+1)\right) / 2^{*}} \varepsilon^{-N}\left(\int_{A^{4 \delta}}\left(\phi(y / \varepsilon) \bar{u}_{\varepsilon}(y / \varepsilon)\right)^{2^{*}} d y\right)^{(p+1) / 2^{*}} \\
& +C\left|A^{4 \delta}\right|^{\left(2^{*}-(p+1)\right) / 2^{*}} \varepsilon^{-N}\left(\int_{A^{4 \delta}}\left(\phi(y / \varepsilon) \bar{v}_{\varepsilon}(y / \varepsilon)\right)^{2^{*}} d y\right)^{(p+1) / 2^{*}} \\
\leq & C\left|A^{4 \delta}\right|^{\left(2^{*}-(p+1)\right) / 2} \varepsilon^{N(p-1) / 2}\left(\int_{\mathbb{R}^{N}}\left|\nabla\left(\phi \bar{u}_{\varepsilon}\right)\right|^{2} d x\right)^{(p+1) / 2} \\
& +C\left|A^{4 \delta}\right|^{\left(2^{*}-(p+1)\right) / 2} \varepsilon^{N(p-1) / 2}\left(\int_{\mathbb{R}^{N}}\left|\nabla\left(\phi \bar{v}_{\varepsilon}\right)\right|^{2} d x\right)^{(p+1) / 2}
\end{aligned}
$$

for some $C>0$ independent of $\varepsilon$. From (45) it follows that

$$
\lim _{\varepsilon \rightarrow 0} \int_{A_{\varepsilon}^{4 \delta}} K(\varepsilon x) Q\left(\bar{u}_{\varepsilon}, \bar{v}_{\varepsilon}\right) d x=0 .
$$

From (8) and the definition of $\chi_{\varepsilon}$, we conclude that

$$
\lim _{\varepsilon \rightarrow 0} \int_{B\left(0, R_{0} / \varepsilon\right) \backslash A_{\varepsilon}^{4 \delta}} K(\varepsilon x) Q\left(\bar{u}_{\varepsilon}, \bar{v}_{\varepsilon}\right) d x=0
$$

and

$$
\lim _{\varepsilon \rightarrow 0} \int_{\mathbb{R}^{N} \backslash B\left(0, R_{0} / \varepsilon\right)} K(\varepsilon x) Q\left(\bar{u}_{\varepsilon}, \bar{v}_{\varepsilon}\right) d x=0 .
$$

As a consequence, from (46), (47) and (48) we have

$$
\lim _{\varepsilon \rightarrow 0} \int_{\mathbb{R}^{N}} K(\varepsilon x) Q\left(\bar{u}_{\varepsilon}, \bar{v}_{\varepsilon}\right) d x=0 .
$$

But this is a contradiction with (7). The proof of lemma is complete.

Completion of the proof for Theorem 1. To complete the proof of Theorem 1, we use arguments developed in [11], [16], [17] and [18]. We define $U_{\varepsilon} \equiv\left(C \alpha_{\varepsilon}\right)^{1 /(p-1)}\left(\bar{u}_{\varepsilon}+\bar{v}_{\varepsilon}\right)$, where $C>0$ was obtained in (37). Note that

$$
-\Delta U_{\varepsilon}+V(\varepsilon x) U_{\varepsilon} \leq K(\varepsilon x)\left(U_{\varepsilon}\right)^{p} \text { in } \mathbb{R}^{N} .
$$

By Lemma 5,

$$
\lim _{\varepsilon \rightarrow 0}\left\|U_{\varepsilon}\right\|_{L^{\infty}\left(\mathbb{R}^{N}\right)}=0
$$

Let

$$
2 c=\inf \left\{V(x) \mid x \in B\left(0,3 R_{0}\right) \backslash Z^{\delta}\right\}>0 .
$$

So, we conclude that

$$
U_{\varepsilon}(x) \leq \exp \left(-c d\left(x, \partial\left(B\left(0,3 R_{0} / \varepsilon\right) \backslash Z_{\varepsilon}^{\delta}\right)\right)\right)
$$

for all $x \in B\left(0,3 R_{0} / \varepsilon\right) \backslash Z_{\varepsilon}^{\delta}$ and for some $c>0$. Indeed, from (49) and (50) it follows that

$$
\Delta U_{\varepsilon}-c U_{\varepsilon} \geq 0 \text { in } B\left(0,3 R_{0} / \varepsilon\right) \backslash Z_{\varepsilon}^{\delta}
$$


for small $\varepsilon>0$. Putting $F_{\varepsilon}(x)=\exp \left(-\sqrt{c} d\left(x, \partial\left(B\left(0,3 R_{0} / \varepsilon\right) \backslash Z_{\varepsilon}^{\delta}\right)\right)\right)$ we deduce that

$$
\Delta F_{\varepsilon}-c F_{\varepsilon}<0 \text { in } B\left(0,3 R_{0} / \varepsilon\right) \backslash Z_{\varepsilon}^{\delta} .
$$

By (50), (52), (53) and the comparison principle we obtain (51). Using (51) we have that

$$
U_{\varepsilon}(x) \leq \exp (-c \delta / \varepsilon) \text { in } Z_{\varepsilon}^{3 \delta} \backslash Z_{\varepsilon}^{2 \delta}
$$

for some constant $c>0$.

For a connected component $\Lambda$ of $\operatorname{int}\left(Z^{4 \delta} \backslash A^{4 \delta}\right)$, we consider the first eigenvalue problem on $\Lambda$,

$$
\left\{\begin{array}{ccc}
-\Delta \phi=\lambda_{1} \phi & \text { in } & \Lambda, \\
\phi=0 & \text { on } & \partial \Lambda .
\end{array}\right.
$$

We can assume that $\max _{x \in \Lambda \cap \partial Z^{3 \delta}} \phi(x) \geq 1$. Now, we claim that

$$
U_{\varepsilon}(x) \leq C \exp (-c \delta / \varepsilon) \text { in } \Lambda_{\varepsilon} \cap Z_{\varepsilon}^{3 \delta}
$$

where $\Lambda_{\varepsilon}=\left\{x \in \mathbb{R}^{N} \mid \varepsilon x \in \Lambda\right\}$. To justify the assertion (56), we define $\phi_{\varepsilon}(x) \equiv \exp (-c \delta / \varepsilon) \phi(\varepsilon x)$. Using $\left(Q_{2}\right)$ and $(8)$, we see that

$$
\int_{\mathbb{R}^{N}} \chi_{\varepsilon}\left(U_{\varepsilon}\right)^{p+1} d x \leq C\left(\alpha_{\varepsilon}\right)^{(p+1) /(p-1)}
$$

for some positive constant $C$. Now, let $z \in Z_{\varepsilon}^{3 \delta} \backslash A_{\varepsilon}^{3 \delta}$. Then, by Lemma 4, (57) and the definition of $\chi_{\varepsilon}$, we conclude that

$$
\int_{B(z, \delta / \varepsilon)}\left(U_{\varepsilon}\right)^{p+1} d x \leq C \varepsilon^{3(p+1) /(p-1)}
$$

for small $\varepsilon>0$. From Theorem 9.20 in [26] and (58) it follows that

$$
\sup _{B(z, \delta / 2 \varepsilon)} U_{\varepsilon} \leq C \varepsilon^{3 /(p-1)}
$$

for small $\varepsilon>0$. Thus,

$$
U_{\varepsilon} \leq C \varepsilon^{3 /(p-1)} \text { in } Z_{\varepsilon}^{3 \delta} \backslash A_{\varepsilon}^{3 \delta} .
$$

From (49) and (59) we have

$$
\Delta U_{\varepsilon}+C_{1} \varepsilon^{3} U_{\varepsilon} \geq 0 \text { in } \Lambda_{\varepsilon} \cap Z_{\varepsilon}^{3 \delta}
$$

for some positive constant $C_{1}$. Since $\phi$ satisfies (55), we deduce that, for small $\varepsilon>0$,

$$
\Delta \phi_{\varepsilon}+C_{1} \varepsilon^{3} \phi_{\varepsilon} \leq 0 \text { in } \Lambda_{\varepsilon} \cap Z_{\varepsilon}^{3 \delta} .
$$

From (54) and the fact that $\phi(x) \geq 1$ for $x \in \Lambda \cap \partial Z^{3 \delta}$, we conclude that $\left(U_{\varepsilon}-\phi_{\varepsilon}\right)_{+}=0$ on $\Lambda_{\varepsilon} \cap\left(Z_{\varepsilon}^{3 \delta} \backslash Z_{\varepsilon}^{2 \delta}\right)$. From (60) and (61) we see that

$$
-\Delta\left(U_{\varepsilon}-\phi_{\varepsilon}\right) \leq C_{1} \varepsilon^{3}\left(U_{\varepsilon}-\phi_{\varepsilon}\right) \text { in } \Lambda_{\varepsilon} \cap Z_{\varepsilon}^{3 \delta} .
$$


As in [11], using $\left(U_{\varepsilon}-\phi_{\varepsilon}\right)_{+}$as a test function in (62) and the Poincaré inequality, we obtain

$$
\begin{aligned}
& \int_{\Lambda_{\varepsilon} \cap Z_{\varepsilon}^{3 \delta}}\left|\nabla\left(U_{\varepsilon}-\phi_{\varepsilon}\right)_{+}\right|^{2} d x \\
\leq & C_{1} \varepsilon^{3} \int_{\Lambda_{\varepsilon} \cap Z_{\varepsilon}^{3 \delta}}\left(\left(U_{\varepsilon}-\phi_{\varepsilon}\right)_{+}\right)^{2} d x \\
\leq & C_{1} \varepsilon^{3}\left(\left|\Lambda_{\varepsilon} \cap Z_{\varepsilon}^{3 \delta}\right| / \omega_{N}\right)^{2 / N} \int_{\Lambda_{\varepsilon} \cap Z_{\varepsilon}^{3 \delta}}\left|\nabla\left(U_{\varepsilon}-\phi_{\varepsilon}\right)_{+}\right|^{2} d x \\
\leq & C \varepsilon \int_{\Lambda_{\varepsilon} \cap Z_{\varepsilon}^{3 \delta}}\left|\nabla\left(U_{\varepsilon}-\phi_{\varepsilon}\right)_{+}\right|^{2} d x
\end{aligned}
$$

for some $C>0$. From (63) it follows that $\left(U_{\varepsilon}-\phi_{\varepsilon}\right)_{+}=0$ in $\Lambda_{\varepsilon} \cap Z_{\varepsilon}^{3 \delta}$ for small $\varepsilon>0$. This shows (56). From (51) and (56), we deduce that for some $C, c>0$,

$$
\left\|U_{\varepsilon}\right\|_{L^{\infty}\left(B\left(0,3 R_{0} / \varepsilon-\delta / \varepsilon\right) \backslash A_{\varepsilon}^{4 \delta}\right)} \leq C \exp (-c \delta / \varepsilon) .
$$

Our next goal is to prove that

$$
U_{\varepsilon}(x) \leq C(\varepsilon /|x|)^{\gamma /(p+1)}
$$

for all $x \in \mathbb{R}^{N} \backslash B\left(0,2 R_{0} / \varepsilon\right)$, where $C>0$ is a constant independent of $y$.

Let $y \in \mathbb{R}^{N} \backslash B\left(0,2 R_{0} / \varepsilon\right)$. From (8), the definition of $\chi_{\varepsilon}$ and of the fact that $\bar{u}_{\varepsilon}$ and $\bar{v}_{\varepsilon}$ are radially symmetric functions, we have

$$
\begin{aligned}
& \int_{B(y, 2)}\left(\alpha_{\varepsilon}\right)^{(p+1) /(p-1)} Q\left(\bar{u}_{\varepsilon}, \bar{v}_{\varepsilon}\right) d x \\
\leq & \frac{C}{|y|^{N-1}} \int_{\left\{x \in \mathbb{R}^{N}|| y|-2 \leq| x|\leq| y \mid+2\right\}}\left(\alpha_{\varepsilon}\right)^{(p+1) /(p-1)} Q\left(\bar{u}_{\varepsilon}, \bar{v}_{\varepsilon}\right) d x \\
\leq & C\left(\varepsilon / R_{0}\right)^{N-1} 2^{\gamma}(\varepsilon /|y|)^{\gamma}\left(\alpha_{\varepsilon}\right)^{(p+1) /(p-1)}
\end{aligned}
$$

for some constant $C>0$. Thus, from $\left(Q_{2}\right),(66)$ and Lemma 4 it follows that

$$
\int_{B(y, 2)}\left(U_{\varepsilon}\right)^{p+1} d x \leq C(\varepsilon /|y|)^{\gamma}
$$

for sufficiently small $\varepsilon>0$ and for some positive constant $C$. Then, from (67) and Theorem 9.20 in [26], we have that

$$
\sup _{B(y, 1)} U_{\varepsilon} \leq C_{0}(\varepsilon /|y|)^{\gamma /(p+1)} \leq C_{1}(\varepsilon /|x|)^{\gamma /(p+1)}
$$

for some constants $C_{0}, C_{1}>0$, for small $\varepsilon>0$ and for any $x \in B(y, 1)$. Hence, (65) follows. We define

$$
\omega_{\varepsilon} \equiv-\frac{(N-2)+\sqrt{(N-2)^{2}+4 \lambda / \varepsilon^{2}}}{2} .
$$


Consequently, $\left(\omega_{\varepsilon}\right)^{2}+(N-2) \omega_{\varepsilon}-\frac{\lambda}{\varepsilon^{2}}=0$. Then, setting $\Psi_{\varepsilon}(x)=|x|^{\omega_{\varepsilon}}$, we conclude from condition $(V)$ that

$$
\begin{aligned}
-\Delta \Psi_{\varepsilon}(x)+V(\varepsilon x) \Psi_{\varepsilon}(x) & \geq\left(2 \lambda / \varepsilon^{2}-\left(\omega_{\varepsilon}\right)^{2}-(N-2) \omega_{\varepsilon}\right) r^{\omega_{\varepsilon}-2} \\
& =\frac{\lambda}{\varepsilon^{2}|x|^{2}} \Psi_{\varepsilon}(x) \text { for }|x| \geq R_{0} / \varepsilon .
\end{aligned}
$$

Using (65), (68) and the fact that $\gamma(p-1) /(p+1)>2$, we have

$$
-\Delta \Psi_{\varepsilon}+V(\varepsilon x) \Psi_{\varepsilon} \geq K(\varepsilon x)\left(U_{\varepsilon}\right)^{p-1} \Psi_{\varepsilon}
$$

for all $x \in \mathbb{R}^{N} \backslash B\left(0,2 R_{0} / \varepsilon\right)$ and small $\varepsilon>0$. From (64), we deduce that for some $C, c>0$,

$$
U_{\varepsilon} \leq C \exp (-c / \varepsilon) \text { in } \partial B\left(0,2 R_{0} / \varepsilon\right) .
$$

Let $\tilde{\Psi}_{\varepsilon}(x)=C \exp (-c / \varepsilon)\left(\frac{2 R_{0}}{\varepsilon}\right)^{-\omega_{\varepsilon}} \Psi_{\varepsilon}(x)$. We claim that

$$
U_{\varepsilon}(x) \leq C \exp (-c / \varepsilon)\left(2 R_{0} / \varepsilon\right)^{-\omega_{\varepsilon}} \Psi_{\varepsilon}(x)
$$

for all $x \in \mathbb{R}^{N} \backslash B\left(0,2 R_{0} / \varepsilon\right)$ and some constants $C, c>0$. In fact, as a consequence of $(70),\left(U_{\varepsilon}-\tilde{\Psi}_{\varepsilon}\right)_{+}=0$ on $\partial B\left(0,2 R_{0} / \varepsilon\right)$. From (69), we note that

$$
-\Delta \tilde{\Psi}_{\varepsilon}+V(\varepsilon x) \tilde{\Psi}_{\varepsilon} \geq K(\varepsilon x)\left(U_{\varepsilon}\right)^{p-1} \tilde{\Psi}_{\varepsilon}
$$

for all $x \in \mathbb{R}^{N} \backslash B\left(0,2 R_{0} / \varepsilon\right)$. As in [11], using (49) and (72) we see that

$$
-\Delta\left(U_{\varepsilon}-\tilde{\Psi}_{\varepsilon}\right)+V(\varepsilon x)\left(U_{\varepsilon}-\tilde{\Psi}_{\varepsilon}\right) \leq\left(U_{\varepsilon}\right)^{p-1} K(\varepsilon x)\left(U_{\varepsilon}-\tilde{\Psi}_{\varepsilon}\right)
$$

for all $x \in \mathbb{R}^{N} \backslash B\left(0,2 R_{0} / \varepsilon\right)$. Multiplying both sides of (73) by $\left(U_{\varepsilon}-\tilde{\Psi}_{\varepsilon}\right)_{+}$and integrating by parts, we obtain

$$
\begin{aligned}
& \int_{\mathbb{R}^{N} \backslash B\left(0,2 R_{0} / \varepsilon\right)}\left[\left|\nabla\left(U_{\varepsilon}-\tilde{\Psi}_{\varepsilon}\right)_{+}\right|^{2}+V(\varepsilon x)\left(\left(U_{\varepsilon}-\tilde{\Psi}_{\varepsilon}\right)_{+}\right)^{2}\right] d x \\
\leq & \int_{\mathbb{R}^{N} \backslash B\left(0,2 R_{0} / \varepsilon\right)}\left(U_{\varepsilon}\right)^{p-1} K(\varepsilon x)\left(\left(U_{\varepsilon}-\tilde{\Psi}_{\varepsilon}\right)_{+}\right)^{2} d x .
\end{aligned}
$$

Using $(V),(65)$ and the fact that $\gamma(p-1) /(p+1)-2>0$, we deduce that, for some constants $C_{0}, C_{1}>0$,

$$
\begin{aligned}
K(\varepsilon x)\left(U_{\varepsilon}(x)\right)^{p-1} & \leq C_{0} \varepsilon^{\gamma(p-1) /(p+1)} \frac{1}{|x|^{\gamma(p-1) /(p+1)-2}} \frac{4 \lambda}{|x|^{2}} \\
& \leq C_{1} \varepsilon^{\gamma(p-1) /(p+1)} V(\varepsilon x)
\end{aligned}
$$

for all $x \in \mathbb{R}^{N} \backslash B\left(0,2 R_{0} / \varepsilon\right)$ and small $\varepsilon>0$. (74) and (75) imply

$$
\begin{aligned}
& \int_{\mathbb{R}^{N} \backslash B\left(0,2 R_{0} / \varepsilon\right)}\left[\left|\nabla\left(U_{\varepsilon}-\tilde{\Psi}_{\varepsilon}\right)_{+}\right|^{2}+V(\varepsilon x)\left(\left(U_{\varepsilon}-\tilde{\Psi}_{\varepsilon}\right)_{+}\right)^{2}\right] d x \\
\leq & C_{1} \varepsilon^{\gamma(p-1) /(p+1)} \int_{\mathbb{R}^{N} \backslash B\left(0,2 R_{0} / \varepsilon\right)} V(\varepsilon x)\left(\left(U_{\varepsilon}-\tilde{\Psi}_{\varepsilon}\right)_{+}\right)^{2} d x .
\end{aligned}
$$

This implies that, for sufficiently small $\varepsilon>0,\left(U_{\varepsilon}-\tilde{\Psi}_{\varepsilon}\right)_{+}=0$ in $\mathbb{R}^{N} \backslash B\left(0,2 R_{0} / \varepsilon\right)$ and the proof of (71) is over. 
Verification of (25). Indeed, from $\left(Q_{2}\right),(64)$ and Lemma 6, we infer that

$$
\begin{aligned}
Q\left(\bar{u}_{\varepsilon}, \bar{v}_{\varepsilon}\right) & \leq 2 \eta_{2}\left(\bar{u}_{\varepsilon}+\bar{v}_{\varepsilon}\right)^{p+1} \\
& =C_{0}\left(\alpha_{\varepsilon}\right)^{-(p+1) /(p-1)}\left(U_{\varepsilon}\right)^{p+1} \\
& \leq C \varepsilon^{-2(p+1) /(p-1)} \exp \left(-c_{1} / \varepsilon\right) \text { in } B\left(0,3 R_{0} / \varepsilon-\delta / \varepsilon\right) \backslash A_{\varepsilon}^{4 \delta}
\end{aligned}
$$

for small $\varepsilon>0$ and for some constants $c_{1}, C_{0}, C>0$. Thus, using the definition of $\chi_{\varepsilon}$ and (76) we see that

$$
\int_{B\left(0, R_{0} / \varepsilon\right) \backslash A_{\varepsilon}^{4 \delta}} \chi_{\varepsilon}(x) Q\left(\bar{u}_{\varepsilon}, \bar{v}_{\varepsilon}\right) d x \leq C_{3} \varepsilon^{-(2 N-1)-5(p+1) /(p-1)} \exp \left(-c_{1} / \varepsilon\right)
$$

and

$$
\int_{B\left(0,2 R_{0} / \varepsilon\right) \backslash B\left(0, R_{0} / \varepsilon\right)} \chi_{\varepsilon}(x) Q\left(\bar{u}_{\varepsilon}, \bar{v}_{\varepsilon}\right) d x \leq C_{4} \varepsilon^{-(2(p+1) /(p-1)+2 \gamma+N)} \exp \left(-c_{1} / \varepsilon\right)
$$

for some constants $C_{3}, C_{4}>0$, independent of $\varepsilon$. Moreover, from $\left(Q_{2}\right)$, Lemma 6 and (71) it follows that, for some constants $C_{5}, c_{2}>0$,

$$
Q\left(\bar{u}_{\varepsilon}, \bar{v}_{\varepsilon}\right) \leq C_{5} \varepsilon^{-2(p+1) /(p-1)} \exp \left(-c_{2} / \varepsilon\right)\left(2 R_{0}\right)^{-(p+1) \omega_{\varepsilon}} \varepsilon^{(p+1) \omega_{\varepsilon}}|x|^{(p+1) \omega_{\varepsilon}}
$$

for all $x \in \mathbb{R}^{N} \backslash B\left(0,2 R_{0} / \varepsilon\right)$ and small $\varepsilon>0$. Then, combining (79) with the definition of $\chi_{\varepsilon}$, we have

$$
\begin{aligned}
& \int_{\mathbb{R}^{N} \backslash B\left(0,2 R_{0} / \varepsilon\right)} \chi_{\varepsilon}(x) Q\left(\bar{u}_{\varepsilon}, \bar{v}_{\varepsilon}\right) d x \\
\leq & C_{5} \exp \left(-c_{2} / \varepsilon\right)\left(2 R_{0}\right)^{-(p+1) \omega_{\varepsilon}} \\
& \times \varepsilon^{-2(p+1) /(p-1)+(p+1) \omega_{\varepsilon}-\gamma} \int_{\mathbb{R}^{N} \backslash B\left(0,2 R_{0} / \varepsilon\right)}|x|^{\gamma+(p+1) \omega_{\varepsilon}} d x \\
(80)= & C_{6} \frac{1}{-\gamma-(p+1) \omega_{\varepsilon}-N}\left(2 R_{0}\right)^{\gamma+N^{-2(p+1) /(p-1)-2 \gamma-N}} \exp \left(-c_{2} / \varepsilon\right)
\end{aligned}
$$

for some constant $C_{6}>0$. From (77), (78), (80) and of the fact that $\chi_{\varepsilon} \equiv 0$ in $A_{\varepsilon}^{4 \delta}$, we deduce that

$$
\lim _{\varepsilon \rightarrow 0} \int_{\mathbb{R}^{N}} \chi_{\varepsilon}(x) Q\left(\bar{u}_{\varepsilon}, \bar{v}_{\varepsilon}\right) d x=0
$$

This proves $(25)$.

As a consequence of (25) we have $\beta_{\varepsilon}=0$. Using (7), the homogeneity of $Q$, Lemma 3 and $\left(\bar{u}_{\varepsilon}, \bar{v}_{\varepsilon}\right)$ as test function in $\left(S_{\alpha_{\varepsilon}, 0}\right)$, we obtain $\frac{M_{\varepsilon}}{p+1}=\alpha_{\varepsilon}$. This implies that $\left(u_{\varepsilon}, v_{\varepsilon}\right)$, where $u_{\varepsilon}(x)=\left(\alpha_{\varepsilon}\right)^{1 /(p-1)} \bar{u}_{\varepsilon}\left(\varepsilon^{-1} x\right)$ and $v_{\varepsilon}(x)=$ $\left(\alpha_{\varepsilon}\right)^{1 /(p-1)} \bar{v}_{\varepsilon}\left(\varepsilon^{-1} x\right)$, satisfies $(S)$. Note that of (64) and (71), we have

$$
u_{\varepsilon}(x), v_{\varepsilon}(x) \leq C \exp (-c \delta / \varepsilon) \forall x \in B\left(0,2 R_{0}\right) \backslash A^{4 \delta}
$$


and

$$
u_{\varepsilon}(x), v_{\varepsilon}(x) \leq C \exp (-c / \varepsilon)\left(|x| / 2 R_{0}\right)^{\omega_{\varepsilon}} \forall x \in \mathbb{R}^{N} \backslash B\left(0,2 R_{0}\right) .
$$

The property (1) is proved in Lemma 5 . We now show the property (2), i.e.,

$$
\liminf _{\varepsilon \rightarrow 0} \varepsilon^{-2 /(p-1)}\left\|u_{\varepsilon}+v_{\varepsilon}\right\|_{L^{\infty}\left(\mathbb{R}^{N}\right)}>0 \text {. }
$$

We define $W_{\varepsilon} \equiv \varepsilon^{-2 /(p-1)}\left(u_{\varepsilon}+v_{\varepsilon}\right)$. Then, it suffices to show that

$$
\lim \inf _{\varepsilon \rightarrow 0}\left\|W_{\varepsilon}\right\|_{L^{\infty}\left(\mathbb{R}^{N}\right)}>0 .
$$

From $\left(Q_{1}\right)$ and of the fact that $\left(u_{\varepsilon}, v_{\varepsilon}\right)$ is solution of $(S)$ we see that, for some $C>0$,

$$
-\Delta W_{\varepsilon}+\frac{1}{\varepsilon^{2}} V(x) W_{\varepsilon} \leq C K(x)\left(W_{\varepsilon}\right)^{p} \text { in } \mathbb{R}^{N} .
$$

Multiplying both sides of (83) by $W_{\varepsilon}$ and integrating by parts, we obtain

$$
\begin{aligned}
& \int_{\mathbb{R}^{N}}\left[\left|\nabla W_{\varepsilon}\right|^{2}+\frac{1}{\varepsilon^{2}} V(x)\left(W_{\varepsilon}\right)^{2}\right] d x \leq C \int_{\mathbb{R}^{N}}\left(W_{\varepsilon}\right)^{p+1} d x \\
\leq & C\left\|W_{\varepsilon}\right\|_{L^{\infty}\left(\mathbb{R}^{N}\right)}^{p-1} \int_{A^{5 \delta}}\left(W_{\varepsilon}\right)^{2} d x+C\left\|W_{\varepsilon}\right\|_{L^{\infty}\left(\mathbb{R}^{N}\right)}^{(p-1) / 2} \int_{\mathbb{R}^{N} \backslash A^{5 \delta}}\left(W_{\varepsilon}\right)^{(p-1) / 2}\left(W_{\varepsilon}\right)^{2} d x .
\end{aligned}
$$

Now, to conclude our proof once more, we will use the arguments developed by Byeon-Wang in [17] and [18]. We take $\varphi \in C_{0}^{\infty}\left(\operatorname{int}\left(A^{5 \delta}\right)\right)$ such that $\varphi(x)=1$ for $x \in A^{4 \delta}$. As $\inf _{x \in \operatorname{supp}(\varphi) \backslash A^{4 \delta}} V(x)>0$ and $\inf _{x \in A^{5 \delta} \backslash A^{4 \delta}} V(x)>0$, it follows, by definition of $\varphi$ and by the Poincaré inequality, that

$$
\begin{aligned}
\int_{A^{5 \delta}}\left(W_{\varepsilon}\right)^{2} d x \leq & 2 \int_{A^{5 \delta}}\left[\left(\varphi W_{\varepsilon}\right)^{2}+(1-\varphi)^{2}\left(W_{\varepsilon}\right)^{2}\right] d x \\
\leq & C_{0} \int_{\operatorname{supp}(\varphi)}\left|\nabla\left(\varphi W_{\varepsilon}\right)\right|^{2} d x+C_{1} \frac{1}{\varepsilon^{2}} \int_{A^{5 \delta} \backslash A^{4 \delta}} V(x)\left(W_{\varepsilon}\right)^{2} d x \\
\leq & C_{2} \frac{1}{\varepsilon^{2}} \int_{\operatorname{supp}(\varphi) \backslash A^{4 \delta}} V(x)\left(W_{\varepsilon}\right)^{2} d x+2 C_{0} \int_{\mathbb{R}^{N}}\left|\nabla W_{\varepsilon}\right|^{2} d x \\
& +C_{1} \frac{1}{\varepsilon^{2}} \int_{A^{5 \delta} \backslash A^{4 \delta}} V(x)\left(W_{\varepsilon}\right)^{2} d x \\
\leq & C \int_{\mathbb{R}^{N}}\left[\left|\nabla W_{\varepsilon}\right|^{2}+\frac{1}{\varepsilon^{2}} V(x)\left(W_{\varepsilon}\right)^{2}\right] d x
\end{aligned}
$$

for some positive constants $C_{0}, C_{1}, C_{2}$ and $C$, independent of small $\varepsilon>0$. On the other hand, using the Hölder inequality and Sobolev embedding results, we get

$$
\int_{\mathbb{R}^{N} \backslash A^{5 \delta}}\left(W_{\varepsilon}\right)^{(p-1) / 2}\left(W_{\varepsilon}\right)^{2} d x \leq C\left(\int_{\mathbb{R}^{N} \backslash A^{5 \delta}}\left(W_{\varepsilon}\right)^{N(p-1) / 4} d x\right)^{2 / N} \int_{\mathbb{R}^{N}}\left|\nabla W_{\varepsilon}\right|^{2} d x .
$$


In view of (81) and (82) we deduce that, for some constants $C_{1}, C_{2}>0$,

$$
W_{\varepsilon}(x) \leq C_{1} \varepsilon^{-2 /(p-1)} \exp (-c \delta / \varepsilon) \forall x \in B\left(0,2 R_{0}\right) \backslash A^{4 \delta}
$$

and

(88) $\quad W_{\varepsilon}(x) \leq C_{2} \varepsilon^{-2 /(p-1)} \exp (-c / \varepsilon)\left(2 R_{0}\right)^{-\omega_{\varepsilon}}|x|^{\omega_{\varepsilon}} \forall x \in \mathbb{R}^{N} \backslash B\left(0,2 R_{0}\right)$.

From (87) and (88) we have, for some constants $c_{1}, c_{2}, C_{3}, C_{4}, C_{5}>0$, that

$$
\begin{aligned}
& \int_{\mathbb{R}^{N} \backslash A^{5 \delta}}\left(W_{\varepsilon}\right)^{N(p-1) / 4} d x \\
\leq & C_{3} \varepsilon^{-N / 2} \exp \left(-c_{1} / \varepsilon\right)+C_{4} \varepsilon^{-N / 2} \exp \left(-c_{2} / \varepsilon\right)\left(2 R_{0}\right)^{-N(p-1) \omega_{\varepsilon} / 4} \\
& \times \int_{\mathbb{R}^{N} \backslash B\left(0,2 R_{0}\right)}|x|^{N(p-1) \omega_{\varepsilon} / 4} d x \\
= & C_{3} \varepsilon^{-N / 2} \exp \left(-c_{1} / \varepsilon\right)+C_{5} \varepsilon^{-N / 2} \exp \left(-c_{2} / \varepsilon\right)\left(2 R_{0}\right)^{N} \frac{1}{-N(p-1) \omega_{\varepsilon} / 4-N} .
\end{aligned}
$$

From (89), $\int_{\mathbb{R}^{N} \backslash A^{5 \delta}}\left(W_{\varepsilon}\right)^{N(p-1) / 4} d x \leq 1$ for sufficiently small $\varepsilon>0$. This and (86) imply

$$
\int_{\mathbb{R}^{N} \backslash A^{5 \delta}}\left(W_{\varepsilon}\right)^{(p-1) / 2}\left(W_{\varepsilon}\right)^{2} d x \leq C \int_{\mathbb{R}^{N}}\left[\left|\nabla W_{\varepsilon}\right|^{2}+\frac{1}{\varepsilon^{2}} V(x)\left(W_{\varepsilon}\right)^{2}\right] d x
$$

for small $\varepsilon>0$. From (84), (85) and (90) it follows that $\left\|W_{\varepsilon}\right\|_{L^{\infty}\left(\mathbb{R}^{N}\right)}^{p-1}+$ $\left\|W_{\varepsilon}\right\|_{L^{\infty}\left(\mathbb{R}^{N}\right)}^{(p-1) / 2} \geq C$ for some positive constant $C$. Then $\left\|W_{\varepsilon}\right\|_{L^{\infty}\left(\mathbb{R}^{N}\right)} \geq C_{1}>0$, where $C_{1}$ is a constant independent of $\varepsilon>0$. This completes the proof of property (2).

Now, we claim that $u_{\varepsilon}, v_{\varepsilon} \in W^{1,2}\left(\mathbb{R}^{N}\right)$. In fact, from (81) and (82) we obtain

$$
\begin{aligned}
\int_{\mathbb{R}^{N}}\left(u_{\varepsilon}\right)^{2} d x \leq & \int_{A^{4 \delta}}\left(u_{\varepsilon}\right)^{2} d x+C_{0} \exp \left(-c_{0} / \varepsilon\right) \\
& +C_{1} \exp \left(-c_{1} / \varepsilon\right)\left(2 R_{0}\right)^{-2 \omega_{\varepsilon}} \int_{\mathbb{R}^{N} \backslash B\left(0,2 R_{0}\right)}|x|^{2 \omega_{\varepsilon}} d x \\
= & \int_{A^{4 \delta}}\left(u_{\varepsilon}\right)^{2} d x+C_{0} \exp \left(-c_{0} / \varepsilon\right) \\
& +C_{2} \exp \left(-c_{1} / \varepsilon\right)\left(2 R_{0}\right)^{N} \frac{1}{-2 \omega_{\varepsilon}-N}
\end{aligned}
$$

for some constants $C_{0}, C_{1}, C_{2}, c_{0}, c_{1}>0$. Using the Lemma 3 and change of variables, we have

$$
\begin{aligned}
\varepsilon^{2} \int_{\mathbb{R}^{N}}\left|\nabla u_{\varepsilon}(x)\right|^{2} d x & =\left(\alpha_{\varepsilon}\right)^{2 /(p-1)} \int_{\mathbb{R}^{N}}\left|\nabla \bar{u}_{\varepsilon}(x / \varepsilon)\right|^{2} d x \\
& =\varepsilon^{N}\left(\alpha_{\varepsilon}\right)^{2 /(p-1)} \int_{\mathbb{R}^{N}}\left|\nabla \bar{u}_{\varepsilon}(y)\right|^{2} d y
\end{aligned}
$$




$$
\begin{aligned}
& \leq \varepsilon^{N}\left(\alpha_{\varepsilon}\right)^{2 /(p-1)}\left\|\left(\bar{u}_{\varepsilon}, \bar{v}_{\varepsilon}\right)\right\|_{\varepsilon}^{2} \\
& =(p+1)\left(\alpha_{\varepsilon}\right)^{(p+1) /(p-1)} \varepsilon^{N} .
\end{aligned}
$$

From (91) and (92) we conclude that $u_{\varepsilon} \in W^{1,2}\left(\mathbb{R}^{N}\right)$. Similarly, we obtain $v_{\varepsilon} \in W^{1,2}\left(\mathbb{R}^{N}\right)$. The decay property (3) follows from (81) and (82).

Acknowledgement. The second author would like to thank the Department of Mathematic of UFMG for their hospitality during his visit.

\section{References}

[1] N. Akhmediev and A. Ankiewicz, Partially coherent solitons on a finite background, Phys. Rev. Lett. 82 (1999), 2661-2664.

[2] C. O. Alves, Local mountain pass for a class of elliptic system, J. Math. Anal. Appl. 335 (2007), no. 1, 135-150.

[3] C. O. Alves and S. H. M. Soares, Existence and concentration of positive solutions for a class gradient systems, Nonlinear Differential Equations Appl. 12 (2005), no. 4, 437-457.

[4] A. Ambrosetti, V. Felli, and A. Malchiodi, Ground states of nonlinear Schrödinger equations with potentials vanishing at infinity, J. Eur. Math. Soc. 7 (2005), no. 1, 117144.

[5] A. Ambrosetti and A. Malchiodi, Perturbation Methods and Semilinear Elliptic Problems on $\mathbb{R}^{N}$, Progr. Math., Birkhäuser 240, Boston, 2006.

[6] A. Ambrosetti, A. Malchiodi, and W.-M. Ni, Singularly perturbed elliptic equations with symmetry: existence of solutions concentrating on spheres, I, Comm. Math. Phys. 235 (2003), no. 3, 427-466.

[7] A. Ambrosetti, A. Malchiodi, and D. Ruiz, Bound states of nonlinear Schrödinger equations with potentials vanishing at infinity, J. Anal. Math. 98 (2006), 317-348.

[8] A. Ambrosetti and P. H. Rabinowitz, Dual variational methods in critical point theory and applications, J. Funct. Anal. 14 (1973), 349-381.

[9] A. Ambrosetti and D. Ruiz, Radial solutions concentrating on spheres of nonlinear Schrödinger equations with vanishing potentials, Proc. Roy. Soc. Edinburgh Sect. A 136 (2006), no. 5, 889-907.

[10] A. Ambrosetti and Z.-Q. Wang, Nonlinear Schrödinger equations with vanishing and decaying potentials, Differential Integral Equations 18 (2005), no. 12, 1321-1332.

[11] M. Badiale, V. Benci, and S. Rolando, A nonlinear elliptic equation with singular potential and applications to nonlinear field equations, J. Eur. Math. Soc. 9 (2007), no. 3 , $355-381$.

[12] M. Badiale and T. d'Aprile, Concentration around a sphere for a singularly perturbed Schrödinger equation, Nonlinear Anal. 49 (2002), no. 7, Ser. A: Theory Methods, 947985.

[13] T. Bartsch and S. Peng, Semiclassical symmetric Schrödinger equations: Existence of solutions concentrating simultaneously on several spheres, Z. Angew. Math. Phys. 58 (2007), no. 5, 778-804.

[14] J. Byeon, Existence of many nonequivalent nonradial positive solutions of semilinear elliptic equations on three-dimensional annuli, J. Differential Equations 136 (1997), no. $1,136-165$.

[15] J. Byeon and L. Jeanjean, Standing waves for nonlinear Schrödinger equations with a general nonlinearity, Arch. Ration. Mech. Anal. 185 (2007), no. 2, 185-200.

[16] J. Byeon and Z.-Q. Wang, Spherical semiclassical states of a critical frequency for Schrödinger equations with decaying potentials, J. Eur. Math. Soc. 8 (2006), no. 2, $217-228$. 
[17] _ Standing waves with a critical frequency for nonlinear Schrödinger equations, Arch. Ration. Mech. Anal. 165 (2002), no. 4, 295-316.

[18] _ Standing waves for nonlinear Schrödinger equations with singular potentials, Ann. Inst. H. Poincaré Anal. Non Linéaire 26 (2009), no. 3, 943-958.

[19] Standing waves with a critical frequency for nonlinear Schrödinger equations. II, Calc. Var. Partial Differential Equations 18 (2003), no. 2, 207-219.

[20] D. N. Christodoulides, T. H. Coskun, M. Mitchell, and M. Segev, Theory of incoherent self-focusing in biased photorefractive media, Phys. Rev. Lett. 78 (1997), 646-649.

[21] R. Cipolatti and W. Zumpichiatti, On the existence and regularity of ground states for a nonlinear system of coupled Schrödinger equations in $\mathbb{R}^{N}$, Comput. Appl. Math. 18 (1999), no. 1, 15-29.

[22] _ Orbitally stable standing waves for a system of coupled nonlinear Schrödinger equations, Nonlinear Anal. 42 (2000), no. 3, Ser. A: Theory Methods, 445-461.

[23] E. N. Dancer and S. Yan, A new type of concentration solutions for a singularly perturbed elliptic problem, Trans. Amer. Math. Soc. 359 (2007), no. 4, 1765-1790.

[24] A. Floer and A. Weinstein, Nonspreading wave packets for the cubic Schrödinger equation with a bounded potential, J. Funct. Anal. 69 (1986), no. 3, 397-408.

[25] B. D. Esry, C. H. Greene, J. P. Burke, Jr., and J. L. Bohn, Hartree-Fock theory for double condensates, Phys. Rev. Lett. 78 (1997), 3594-3597.

[26] D. Gilbarg and N. S. Trudinger, Elliptic Partial Differential Equations of Second Order, 2nd ed., Grundlehren Math. 224, Springer-Verlag, Berlin Heidelberg, 1983.

[27] A. Hasegawa and Y. Kodama, Solitions in Optical Communications, Academic Press, San Diego, 1995.

[28] M. N. Islam, Ultrafast Fiber Switching Devices and Systems, Cambridge University Press, New York, 1992.

[29] I. P. Kaminow, Polarization in optical fibers, IEEE J. Quantum Electron. 17 (1981), $15-22$.

[30] E. H. Lieb and R. Seiringer, Proof of Bose-Einstein condensation for dilute trapped gases, Phys. Rev. Lett. 88 (2002), 170409.

[31] L. A. Maia, E. Montefusco, and B. Pellacci, Positive solutions for a weakly coupled nonlinear Schrödinger system, J. Differential Equations 229 (2006), no. 2, 743-767.

[32] C. R. Menyuk, Nonlinear pulse propagation in birefringence optical fiber, IEEE J. Quantum Electron. 23 (1987), 174-176.

[33] _ Pulse propagation in an elliptically birefringent Kerr medium, IEEE J. Quantum Electron. 25 (1989), 2674-2682.

[34] P. Meystre, Atom Optics, Springer-Verlag, New York, 2001.

[35] D. L. Mills, Nonlinear Optics, Springer-Verlag, Berlin, 1998.

[36] D. C. de Morais Filho and M. A. S. Souto, Systems of p-laplacean equations involving homogeneous nonlinearities with critical Sobolev exponent degrees, Comm. Partial Differential Equations 24 (1999), no. 7-8, 1537-1553.

[37] A. Pomponio, Coupled nonlinear Schrödinger systems with potentials, J. Differential Equations 227 (2006), no. 1, 258-281.

Paulo Cesar Carrião

Departamento de Matemática

Universidade Federal de Minas Gerais

31270-010 Belo Horizonte(MG), Brazil

E-mail address: carrion@mat.ufmg.br 
NARCiso Horta Lisboa

Departamento de Ciências Exatas

Universidade Estadual de Montes Claros

39401-089 Montes Claros(MG), Brazil

E-mail address: narciso.lisboa@unimontes.br

Olimpio Hiroshi MiYagaki

Departamento de Matemática

Universidade Federal de Juiz de Fora

36036-330 JuIz de ForA(MG), BraziL

E-mail address: ohmiyagaki@gmail.com 